\title{
Adaptive Fast Nonsingular Fixed-Time Tracking Control for Robot Manipulators
}

\author{
Huihui Pan $\mathbb{D}^{1,2}$ and Guangming Zhang $\mathbb{D}^{1}$ \\ ${ }^{1}$ School of Mechanical and Power Engineering, Nanjing Tech University, Nanjing 211816, China \\ ${ }^{2}$ College of Electrical Engineering, Tongling University, Tongling 244061, China
}

Correspondence should be addressed to Guangming Zhang; zgm@njtech.edu.cn

Received 29 November 2020; Accepted 24 April 2021; Published 8 May 2021

Academic Editor: Guang Li

Copyright ( $\odot 2021$ Huihui Pan and Guangming Zhang. This is an open access article distributed under the Creative Commons Attribution License, which permits unrestricted use, distribution, and reproduction in any medium, provided the original work is properly cited.

\begin{abstract}
This paper studies the fixed-time trajectory tracking control problem of robot manipulators in the presence of uncertain dynamics and external disturbances. First, a novel nonsingular fixed-time sliding mode surface is presented, which can ensure that the convergence time of the suggested surface is bounded regardless of the initial states. Subsequently, a novel fast nonsingular fixedtime sliding mode control (NFNFSMC) is developed so that the closed-loop system is fixed-time convergent to the equilibrium. By applying the proposed NFNFSMC method and the adaptive technique, a novel adaptive nonsingular fixed-time control scheme is proposed, which can guarantee fast fixed-time convergence of the tracking errors to small regions around the origin. With the proposed control method, the lumped disturbance is compensated by the adaptive technique, whose prior information about the upper bound is not needed. The fixed-time stability of the trajectory tracking control under the proposed controller is proved by the Lyapunov stability theory. Finally, corresponding simulations are given to illustrate the validity and superiority of the proposed control approach.
\end{abstract}

\section{Introduction}

In the past decades, many research efforts [1-6] have been devoted to addressing the trajectory tracking problem of robot manipulators in the presence of uncertain dynamics and external disturbances. Several advanced control strategies have been employed in robot manipulator control systems, such as sliding mode control (SMC) [7-11], feedback linearization [12], backstepping control [13], neural network control [14], and model predictive control [15]. Among them, SMC is a popular method owing to its fast convergence, insensitivity property to uncertain dynamics, and strong robustness to external disturbances [16-18].

Among the SMC category, terminal sliding mode control (TSMC) $[19,20]$ can achieve the robust finite-time tracking of robot manipulators. However, standard TSMC may cause the singularity problem [21]. To remedy the singularity of the TSMC, many effective methods have been put forward. One method $[22,23]$ to overcome the singularity problem was given by developing a new type of TSM without singularity known as nonsingular TSM (NTSM). Another method [24] to avoid the singularity was presented by switching the terminal sliding mode surface to a general sliding mode surface with a nonlinear function. Additionally, fast nonsingular terminal sliding mode control (FNTSMC) has been widely investigated for the robot manipulator control system to enhance the convergence rate [25-28]. Reference [25] proposed a new fast nonsingular terminal sliding mode manifold combining the satisfactory characteristics of the linear SM and the NTSM to achieve fast finite-time stable tracking. A FNTSMC scheme with the adaptive technique was presented for robot manipulators to ensure that the position tracking errors could converge to zero within finite time in [26]. In [27], an improved NTSMC based on a nonlinear function was proposed for robot manipulators, which could guarantee system performance and fast finite- 
time stability. A novel adaptive second-order FNTSMC was introduced to achieve fast finite-time convergence and good tracking precision in [28]. Because both the sliding variables and the tracking errors can be stabilized to the equilibrium in finite time, the aforementioned SMCs are known as the finite-time controls.

These finite-time controls have a drawback that the convergence time is related to the initial conditions of robotic systems. That is to say, the settling time of trajectory tracking cannot be acquired priorly. Recently, as an extension of the finite-time control, the fixed-time control has received a lot of attention [29-31]. Compared with the finitetime control, the fixed-time control can ensure that the convergence time is upper bounded by a fixed time and independent of initial conditions. The fixed-time control has been widely employed in many nonlinear control systems. More specifically, [32] presented a novel fixed-time output feedback control which could be employed to double integrator systems. In [33], an adaptive nonsingular fixed-time control strategy was proposed for the tracking control of the rigid spacecraft, which could guarantee the fixed-time stability of both the attitude and angular velocity. In [34], an adaptive fast nonsingular terminal sliding mode guidance law was designed, which could achieve system stabilization within a fixed time. A novel fixed-time NTSMC method was applied to a single inverted pendulum control system in [35]. Reference [30] developed a fixed-time convergent guidance law with impact angle control so that the impact angle error could be stabilized to zero before the interception within a fixed time. The above literature review indicates that the fixed-time controls are applicable to some physical control systems and can obtain system stabilization with fixed-time convergence. As far as the authors know, little attention has been paid to the fixed-time tracking control of uncertain robot manipulators. Moreover, further accelerating the convergence rate of tracking control is worth being considered in the controller design.

This paper focuses on the development of an adaptive fast nonsingular fixed-time tracking control for uncertain robot manipulators so that satisfactory features including fast fixed-time convergence and high steady-state tracking precision are provided. The main contributions can be summarized as follows:

(1) A novel fixed-time nonsingular fast terminal sliding mode manifold (NFNFSM) is developed to shorten the time during which the system states arrive at the equilibrium.

(2) Based on the proposed NFNFSM, a novel fixed-time nonsingular fast terminal sliding mode controller (NFNFSMC) is designed. Moreover, the proof of fixed-time stability is provided in detail.

(3) An adaptive NFNFSMC (ANFNFSMC) scheme is presented by combining the proposed NFNFSMC and the adaptive technique. The lumped disturbance is compensated by the designed adaptive law, whose prior information about the upper bound is not needed. The proposed ANFNFSMC can not only obtain strong robustness to uncertain disturbances but also achieve fast fixed-time convergence of robot manipulator systems.

The rest of this paper is organized as follows: Section 2 provides the dynamic model of robot manipulators and some lemmas. The NFNFSMC algorithm is presented in Section 3. In Section 4, the ANFNFSMC strategy is proposed for the tracking control of robot manipulators. Simulation results illustrate the feasibility and superiority of the proposed control scheme in Section 5. Finally, the conclusion is shown in Section 6.

Throughout the paper, $\operatorname{sig}(x)^{\alpha}$ denotes $|x|^{\alpha} \operatorname{sign}(x)$.

\section{Mathematical Preliminaries}

2.1. Model of Robot Manipulators. The dynamic equation of the n-link robot manipulator model with uncertain disturbances can be described as [1]

$$
M(q) \ddot{q}+C(q, \dot{q}) \dot{q}+G(q)=u+d,
$$

whereq, $\dot{q}, \ddot{q} \in R^{n}$ stand for the vectors of joint positions, velocities, and accelerations, respectively. $M(q)$ is the positive definite inertia matrix, $C(q, \dot{q})$ is the centripetal Coriolis matrix, and $G(q)$ is the gravitational vector. $u$ is the control input vector and $d$ is the external disturbance vector.

Assumption 1. The model parameters in (1) are given by

$$
\begin{aligned}
M(q) & =M_{0}(q)+\Delta M(q), \\
C(q, \dot{q}) & =C_{0}(q, \dot{q})+\Delta C(q, \dot{q}), \\
G(q) & =G_{0}(q)+\Delta G(q),
\end{aligned}
$$

where $M_{0}(q), C_{0}(q, \dot{q})$, and $G_{0}(q)$ represent the nominal terms. $\Delta M(q), \Delta C(q, \dot{q})$, and $\Delta G(q)$ represent the uncertain terms.

According to Assumption 1, system (1) can be expressed as follows:

$$
M_{0}(q) \ddot{q}+C_{0}(q, \dot{q}) \dot{q}+G_{0}(q)=u+\rho,
$$

in which the lumped disturbance $\rho$ is defined as

$$
\rho=-\Delta M(q) \ddot{q}-\Delta C(q, \dot{q}) \dot{q}-\Delta G(q)+d .
$$

Assumption 2. The lumped disturbance $\rho$ is bounded by

$$
\|\rho\|<a_{0}+a_{1}\|q\|+a_{2}\|\dot{q}\|^{2},
$$

where $a_{0}, a_{1}$, and $a_{2}$ are unknown positive constants.

Assumption 3. The norms of desired vectors are bounded by

$$
\begin{gathered}
\left\|q_{d}\right\| \leq b_{0}, \\
\left\|\dot{q}_{d}\right\| \leq b_{1},
\end{gathered}
$$

where $q_{d}$ and $\dot{q}_{d}$ denote the vector of desired position and velocity, respectively. $b_{0}$ and $b_{1}$ are positive constants.

The research focus of this paper is to propose a novel fast nonsingular fixed-time sliding mode control strategy for robot manipulators in the existence of uncertainties and 
disturbances such that fast fixed-time stability can be guaranteed.

\subsection{Fundamental Facts}

Definition 1 (see [36]). Consider a dynamic system

$$
\begin{aligned}
\dot{z} & =f(z), \\
z(0) & =z_{0}, \\
f(0) & =0,
\end{aligned}
$$

where $z \in R^{n}, f(z): D \in R^{n}$ is a continuous nonlinear function that is an open neighborhood $D$ of the origin. The system is fixed-time stable if the convergence time is a bounded function $T\left(z_{0}\right)$, that is, there exists a time constant $T_{\max }$ such that $T\left(z_{0}\right)<T_{\max }$.

Lemma 1 (see [37]). If system (7) is fixed-time stable, then there exists a continuous positive Lyapunov function $V(z)$, $\alpha, \beta, a, b \in R^{+}, a<1, b>1$, and $0<\mathrm{H}<\infty$ satisfying that $\dot{V}(z) \leq-\alpha V(z)^{a}-\beta V(z)^{b}+H$. Then, the system can reach the residual set which is bounded as $\left\{\lim _{\text {. }}\right.$ $\left.T z \mid V(z) \leq \min \left\{[H /(\alpha(1-\theta))]^{1 / a},[H /(\beta(1-\quad \theta))]^{1 / b}\right\}\right\}$, where $\theta$ is scalar and satisfies $0<\theta \leq 1$. The time to reach the neighborhood of the origin is upper bounded by $T \leq 1 /(\alpha \theta(1-a))+1 /(\beta \theta(b-1))$.

Lemma 2 (see $[38,39])$. For any nonnegative real numbers $\zeta_{1}, \zeta_{2}, \ldots \zeta_{n}, p>1$, and $0<q \leq 1$, the following two inequalities hold:

$$
\begin{aligned}
& \sum_{i=1}^{n} \zeta_{i}^{p} \geq n^{1-p}\left(\sum_{i=1}^{n} \zeta_{i}\right)^{p}, \\
& \sum_{i=1}^{n} \zeta_{i}^{q} \geq\left(\sum_{i=1}^{n} \zeta_{i}\right)^{q} .
\end{aligned}
$$

Lemma 3. Consider the following nonlinear system:

$$
\dot{z}=-\alpha \operatorname{sig}(z)^{\gamma}-c \operatorname{sig}(z)^{\eta},
$$

where $\alpha>0, c>0, \gamma=0.5(r+1)+0.5(r-1) \operatorname{sign}(|z|-1)$, $\eta=0.5(r+\varphi)+0.5(r-\varphi) \operatorname{sign}(|z|-1), \quad r>1, \quad 0<\varphi<1$. System (9) is fixed-time stable with the convergence time $T$ bounded by

$$
T \leq T_{\max }=\frac{1}{(\alpha+c)(r-1)}+\frac{1}{\alpha(1-\varphi)} \ln \left(1+\frac{\alpha}{c}\right) .
$$

Proof. The differential equation for system (9) can be converted into the following form:

$$
\begin{cases}\dot{z}=-\alpha \operatorname{sig}(z)^{r}-c \operatorname{sig}(z)^{r}, & |z|>1, \\ \dot{z}=-\alpha z-c \operatorname{sig}(z)^{\varphi}, & |z| \leq 1 .\end{cases}
$$

Denote $\quad \chi=1+\operatorname{In}|z|$ for $|z|>1$. Denote $\quad \chi=|z|^{1-\varphi}$ for $|z| \leq 1$. The initial values of $z$ and $\chi$ are defined as $z_{0}$ and $\chi_{0}$. Equation (11) can be rewritten as

$$
\begin{cases}\dot{\chi}=-\alpha e^{(r-1)(\chi-1)}-c e^{(r-1)(\chi-1)}, & \chi>1, \\ \dot{\chi}=-\alpha \chi(1-\varphi)-c(1-\varphi), & 0<\chi \leq 1 .\end{cases}
$$

Solving (12), the upper bound of convergence time can be calculated as

$$
\begin{aligned}
T_{\max } & =\lim _{z_{0} \longrightarrow \infty} T\left(z_{0}\right) \\
& =\lim _{\chi_{0} \longrightarrow \infty}\left(\int_{1}^{\chi_{0}} \frac{1}{(\alpha+c) e^{(r-1)(\chi-1)}} \mathrm{d} \chi+\int_{0}^{1} \frac{1}{(1-\varphi)(\alpha \chi+c)} \mathrm{d} \chi\right) \\
& =\lim _{\chi_{0} \rightarrow \infty} \int_{1}^{\chi_{0}} \frac{1}{(\alpha+c) e^{(r-1)(\chi-1)}} \mathrm{d} \chi+\frac{1}{\alpha(1-\varphi)} \ln \left(1+\frac{\alpha}{c}\right) .
\end{aligned}
$$

Define $\xi=e^{(r-1)(\chi-1)}$ and denote $\xi_{0}$ as the initial value of $\xi$; we have

$$
\begin{aligned}
& \lim _{\chi_{0} \longrightarrow \infty} \int_{1}^{\chi_{0}} \frac{1}{(\alpha+c) e^{(r-1)(\chi-1)}} \mathrm{d} \chi \\
& =\frac{1}{r-1} \lim _{\xi_{0} \longrightarrow \infty} \int_{1}^{\xi_{0}} \frac{1}{\xi(\alpha \xi+c \xi)} \mathrm{d} \xi \\
& =\frac{1}{(\alpha+c)(r-1)} .
\end{aligned}
$$

Thus, for system (9), the upper bound of convergence time can be expressed as

$$
T_{\max }=\frac{1}{(\alpha+c)(r-1)}+\frac{1}{\alpha(1-\varphi)} \ln \left(1+\frac{\alpha}{c}\right) .
$$

The proof of Lemma 3 is completed.

Remark 1. Reference [40] presented a fixed-time stable system $\dot{z}=-\alpha \operatorname{sig}(z)^{r}-c \operatorname{sig}(z)^{\varphi}$, and the system can be stabilized within a fixed time $T<1 /(\alpha(r-1))+1 /(c(1-\varphi))$. A fast fixed-time stable system $\dot{z}=-\alpha \operatorname{sig}(z)^{\gamma}-c \operatorname{sig}(z)^{\varphi}$ with $\gamma=0.5(r+1)+0.5(r-1) \operatorname{sign}(|z|-1)$ was introduced in [35], and the system can be stabilized within a fixed time $T<1 /(\alpha(r-1))+\ln (1+\alpha / c) /(\alpha(1-\varphi))$. Since the relations $1 /(\alpha+c)<1 / \alpha$ and $\ln (1+\alpha / c)<\alpha / c$ hold, the proposed system (9) can offer a fast convergence rate than the systems presented in $[35,40]$.

\section{Novel Nonsingular Fixed-Time Control and Stability Analysis}

In this section, a novel nonsingular fixed-time control and the related stability analysis are presented.

3.1. Novel Nonsingular Fixed-Time Control. For a clear interpretation of the key idea, we first consider the novel nonsingular fixed-time control of a single second-order system given by 


$$
\begin{aligned}
& \dot{x}_{1}=x_{2}, \\
& \dot{x}_{2}=u,
\end{aligned}
$$

where $x_{1}$ and $x_{2}$ are system states. $u$ is the control input.

Based on Lemma 3, for system (16), a new form of fixedtime sliding mode surface is designed as

$$
s=x_{2}+\alpha_{1} \operatorname{sig}\left(x_{1}\right)^{\gamma_{1}}+c_{1} \operatorname{sig}\left(x_{1}\right)^{\eta_{1}},
$$

where $r_{1}>1, \quad 0<\varphi_{1}<1, \quad \gamma_{1}=0.5\left(r_{1}+1\right)+0.5\left(r_{1}-1\right)$ $\operatorname{sign}\left(\left|x_{1}\right|-1\right), \eta_{1}=0.5\left(r_{1}+\varphi_{1}\right)+0.5\left(r_{1}-\varphi_{1}\right) \operatorname{sign}\left(\left|x_{1}\right|-1\right)$.

Differentiating (17) results in

$$
\dot{s}=\dot{x}_{2}+\alpha_{1} \gamma_{1}\left|x_{1}\right|^{\gamma_{1}-1} x_{2}+c_{1} \eta_{1}\left|x_{1}\right|^{\eta_{1}-1} x_{2} .
$$

For (18), if $x_{1}=0$ and $x_{2} \neq 0$, it may suffer from the singularity problem due to $\varphi_{1}-1<0$.

To circumvent the singularity problem, a novel fast nonsingular fixed-time sliding mode surface (NFNFSM) is proposed as

$$
s=x_{1}+\beta \operatorname{sig}\left(\alpha_{1} \operatorname{sig}\left(x_{1}\right)^{\gamma_{1}}+x_{2}\right)^{1 / \eta_{1}},
$$

where $\alpha_{1}>0, c_{1}>0, r_{1}>1,0<\varphi_{1}<1, \beta=c_{1}^{-1 / \eta_{1}}$,

$$
\begin{aligned}
& \gamma_{1}=0.5\left(r_{1}+1\right)+0.5\left(r_{1}-1\right) \operatorname{sign}\left(\left|x_{1}\right|-1\right), \\
& \eta_{1}=0.5\left(r_{1}+\varphi_{1}\right)+0.5\left(r_{1}-\varphi_{1}\right) \operatorname{sign}\left(\frac{\left|\alpha_{1} \operatorname{sig}\left(x_{1}\right)^{\gamma_{1}}+x_{2}\right|}{c_{1}}-1\right) .
\end{aligned}
$$

Remark 2. When $s=0$ is achieved, it can be deduced that $x_{2}=-\alpha_{1} \operatorname{sig}\left(x_{1}\right)^{\gamma_{1}}-c_{1} \operatorname{sig}\left(x_{1}\right)^{\eta_{1}}$ and $\left|x_{1}\right|^{\eta_{1}}=\mid \alpha_{1} \operatorname{sig}\left(x_{1}\right)^{\gamma_{1}}$ $+x_{2} \mid / c_{1}$.

When $\left|\alpha_{1} \operatorname{sig}\left(x_{1}\right)^{\gamma_{1}}+x_{2}\right| / c_{1} \geq 1$, then $\eta_{1}=r_{1}$ and $\left|x_{1}\right| \geq 1$. When $\left|\alpha_{1} \operatorname{sig}\left(x_{1}\right)^{\gamma_{1}}+x_{2}\right| / c_{1}<1$, then $\eta_{1}=\varphi_{1}$ and $\left|x_{1}\right|<1$. Therefore, onces $=0$ is satisfied, $\eta_{1}$ is equivalent to the equality $\eta_{1}=0.5\left(r_{1}+\varphi_{1}\right)+0.5\left(r_{1}-\varphi_{1}\right) \operatorname{sign}\left(\left|x_{1}\right|-1\right)$.
Remark 3. Note that the proposed NFNFSM can solve the singularity problem without switching the terminal sliding mode surface into a general sliding mode surface, which is different from some existing nonsingular fixed-time sliding mode manifolds, such as in [24, 27].

Next, to illustrate the superiority of the proposed sliding surface, the convergence performance of NFSM in [39], FNFSM in [34], and the proposed NFNFSM are compared in the sliding motion. The sliding surface NFSM [39] is

$$
s=x_{1}+\operatorname{sig}\left(k x_{2}\right)^{q / p},
$$

with

$$
k=\frac{1}{\alpha_{1}\left|x_{1}\right|^{m / n-p / q}+c_{1}},
$$

where $\alpha_{1}, c_{1}>0$ and $m, n, p$, and $q$ are odd integers satisfying that $p<q<2 p, m / n-p / q>1$.

The sliding surface FNFSM [34] is

$$
s=x_{2}+\alpha_{1} \operatorname{sig}\left(x_{1}\right)^{\gamma_{1}}+c_{1} \mu\left(x_{1}\right)
$$

with

$$
\mu\left(x_{1}\right)= \begin{cases}\operatorname{sig}\left(x_{1}\right)^{\varphi_{1}}, & \left|x_{1}\right| \geq \delta, \\ \beta_{1} x_{1}+\beta_{2} \operatorname{sig}\left(x_{1}\right)^{2}, & \left|x_{1}\right|<\delta,\end{cases}
$$

where $\gamma_{1}=0.5(r+1)+0.5(r-1) \operatorname{sign}\left(\left|x_{1}\right|-1\right), \quad 0<\delta<1$, $\beta_{1}=\left(2-\varphi_{1}\right) \delta^{\varphi_{1}-1}, \beta_{2}=\left(\varphi_{1}-1\right) \delta^{\varphi_{1}-2}$.

The parameters of the three sliding surfaces are selected as $\alpha_{1}=c_{1}=1, r=2, \varphi_{1}=0.6, m / n=2, p / q=0.6, \delta=0.01$.

The same initial condition is that $x_{1}(0)=5$. We illustrate the convergence of the three sliding surfaces in Figure 1. It can be noted from Figure 1 that the proposed NFNFSM offers a faster convergence rate than NFSM and FNFSM.

Based on the proposed sliding surface (19), a novel fixedtime controller is designed as

$$
\begin{aligned}
u= & -\left[\frac{\eta_{1}}{\beta} \operatorname{sig}\left(\alpha_{1} \operatorname{sig}\left(x_{1}\right)^{\gamma_{1}}+x_{2}\right)^{2-\left(1 / \eta_{1}\right)}+\alpha_{1} \gamma_{1}\left|x_{1}\right|^{\gamma_{1}-1} x_{2}\right. \\
& \left.+\alpha_{1} \eta_{1}\left|x_{1}\right|^{\gamma_{1}-1}\left(\alpha_{1} \operatorname{sig}\left(x_{1}\right)^{\gamma_{1}}+x_{2}\right)+\frac{\eta_{1}}{\beta}\left|\alpha_{1} \operatorname{sig}\left(x_{1}\right)^{\gamma_{1}}+x_{2}\right|^{1-\left(1 / \eta_{1}\right)} \cdot \psi_{\varepsilon}\left(\left|\alpha_{1} \operatorname{sig}\left(x_{1}\right)^{\gamma_{1}}+x_{2}\right|\left(1 / \eta_{1}\right)-1\right) \cdot\left(\alpha_{2} \operatorname{sig}(s)^{r_{2}}+c_{2} \operatorname{sig}(s)^{\varphi_{2}}\right)\right],
\end{aligned}
$$

in which $\psi_{\varepsilon}(\cdot):[0,+\infty) \longrightarrow[0,1]$ as

$$
\psi_{\varepsilon}(x)= \begin{cases}\sin \left(\frac{\pi}{2} \cdot \frac{x}{\varepsilon}\right), & x \leq \varepsilon, \\ 1, & x>\varepsilon,\end{cases}
$$

where $\alpha_{2}>0, c_{2}>0, r_{2}>1,0<\varphi_{2}<1, x>0,0<\varepsilon<1$, and $\psi_{\varepsilon}(x) / x \longrightarrow \pi /(2 \varepsilon)$ as $x \longrightarrow 0$.

\subsection{Stability Analysis}

Theorem 1. Consider system (16) with the proposed fixedtime controller defined by (25). Then the system states can converge to the origin within a fixed time and the convergence time is expressed as

$$
T<T_{\max }=T_{1}+T_{2}+\phi(\varepsilon),
$$


where

$$
\begin{aligned}
& T_{1}=\frac{1}{\left(\alpha_{2}\left(r_{2}-1\right)\right)}+\frac{1}{\left(c_{2}\left(1-\varphi_{2}\right)\right)} \\
& T_{2}=\frac{1}{\left(\left(\alpha_{1}+c_{1}\right)\left(r_{1}-1\right)\right)}+\frac{\ln \left(1+\alpha_{1} / c_{1}\right)}{\left(\alpha_{1}\left(1-\varphi_{1}\right)\right)},
\end{aligned}
$$

and $\phi(\varepsilon)$ represents a small time margin associated with $\varepsilon$.

Proof. Select the following Lyapunov function:

$$
V_{1}=0.5 s^{2}
$$

The derivative of $V_{1}$ to time is

$$
\dot{V}_{1}=s\left(x_{2}+\frac{\beta}{\eta_{1}}\left|\alpha_{1} \operatorname{sig}\left(x_{1}\right)^{\gamma_{1}}+x_{2}\right|^{\left(1 / \eta_{1}\right)-1}\left(\alpha_{1} \gamma_{1}\left|x_{1}\right|^{\gamma_{1}-1} x_{2}+\dot{x}_{2}\right)\right) \text {. }
$$

Substituting (25) into (30), there is

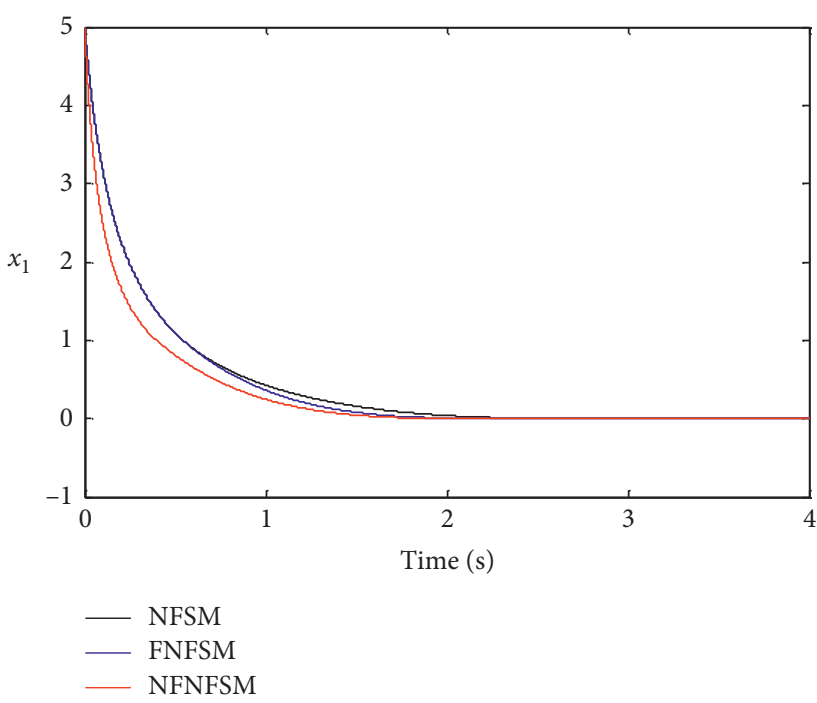

FIgURE 1: Comparison of the three sliding surfaces.

$$
\begin{aligned}
\dot{V}_{1} & =-s \psi_{\varepsilon}\left(\left|\alpha_{1} \operatorname{sig}\left(x_{1}\right)^{\gamma_{1}}+x_{2}\right|^{\left(1 / \eta_{1}\right)-1}\right)\left(\alpha_{2} \operatorname{sig}(s)^{r_{2}}+c_{2} \operatorname{sig}(s)^{\varphi_{2}}\right)-s \alpha_{1}\left|x_{1}\right|^{\gamma_{1}-1} s \\
& \leq-\psi_{\varepsilon}\left(\left|\alpha_{1} \operatorname{sig}\left(x_{1}\right)^{\gamma_{1}}+x_{2}\right|^{\left(1 / \eta_{1}\right)-1}\right)\left(\alpha_{2}|s|^{r_{2}+1}+c_{2}|s|^{\varphi_{2}+1}\right) \\
& =-\psi_{\varepsilon}\left(\left|\alpha_{1} \operatorname{sig}\left(x_{1}\right)^{\gamma_{1}}+x_{2}\right|^{\left(1 / \eta_{1}\right)-1}\right)\left(\alpha_{2}\left(2 V_{1}\right)^{\left(r_{2}+1\right) / 2}+c_{2}\left(2 V_{1}\right)^{\left(\varphi_{2}+1\right) / 2}\right) .
\end{aligned}
$$

Denote $\omega=\left|\alpha_{1} \operatorname{sig}\left(x_{1}\right)^{\gamma_{1}}+x_{2}\right|^{1 / \eta_{1}-1}, \quad g=\alpha_{1} \operatorname{sig}\left(x_{1}\right)^{\gamma_{1}}+$ $x_{2}$. According to $(31), \psi_{\varepsilon}(\omega)>0$ if $g \neq 0$. To facilitate analysis, the state space $x \in R^{2}$ is divided into two separate regions as $\Omega_{1}=\left\{\left.\left(x_{1}, x_{2}\right)|\omega=| g\right|^{1 / \eta_{1}-1} \geq \varepsilon\right\}, \Omega_{2}=\left\{\left.\left(x_{1}, x_{2}\right)|\omega=| g\right|^{1 / \eta_{1}-1}\right.$ $<\varepsilon\}$.

Case 1. When the states $\left(x_{1}, x_{2}\right)$ enter into the region $\Omega_{1}$, then the function $\psi_{\varepsilon}(\omega)=1$. If $V_{1} \neq 0$, let $y_{0}=\sqrt{2 V_{1}}$ and $\dot{y}_{0}=\dot{V}_{1} / \sqrt{2 V_{1}}$, and we obtain $\dot{y}_{0} \leq-\alpha_{2}$ sig $\left(y_{0}\right)^{r_{2}}-c_{2} \operatorname{sig}\left(y_{0}\right)^{\varphi_{2}}$. According to [39], the states $\left(x_{1}, x_{2}\right)$ will arrive at the sliding surface $s=0$ or enter into the region $\Omega_{2}$ within fixed time $T_{1}$.

Case 2. In the region $\Omega_{2}, 0<\psi_{\varepsilon}(\Phi)<1$ if $g \neq 0$. It can be deduced from (31) that $s=0$ is still attractive. Next, we need to prove that $g=0$ is not attractive except for the origin $\left(x_{1}, x_{2}\right)=(0,0)$. When $g$ is very close to 0 , the control law (25) reduces to the following form:

$$
u=-\alpha_{1} \gamma_{1}\left|x_{1}\right|^{\gamma_{1}-1} x_{2}-\frac{\pi \eta_{1}}{2 \beta \varepsilon}\left(\alpha_{2} \operatorname{sig}(s)^{r_{2}}+c_{2} \operatorname{sig}(s)^{\varphi_{2}}\right),
$$

where the fact $\psi_{\varepsilon}(\bowtie) / \Phi \longrightarrow \pi /(2 \varepsilon)$, as $g \longrightarrow 0$ is used.

Differentiating $g$ and combining (32), we have

$$
\dot{g}=\alpha_{1} \gamma_{1}\left|x_{1}\right|^{\gamma_{1}-1} x_{2}+\dot{x}_{2}=-\frac{\pi \eta_{1}}{2 \beta \varepsilon}\left(\alpha_{2} \operatorname{sig}(s)^{r_{2}}+c_{2} \operatorname{sig}(s)^{\varphi_{2}}\right) .
$$

From (33), it can be concluded that $\dot{g}<0$ for $s>0$ and $\dot{g}>0$ for $s<0$. This means that $g=0$ is not an attractor. Then, the system states $\left(x_{1}, x_{2}\right)$ will transgress $\Omega_{2}$ into $\Omega_{1}$ monotonically in a small time margin $\phi(\varepsilon)$.

Accordingly, the sliding surface $s=0$ can be arrived at within time $T_{r}<T_{1}+\phi(\varepsilon)$. Once the sliding surface $s=0$ is reached, it can be known from Lemma 3 that the system states $\left(x_{1}, x_{2}\right)$ can converge to the origin within fixed time $T_{s}<T_{2}$. Then, the total convergence time is upper bounded by (27).

The proof of Theorem 1 is completed.

Remark 4. Note that the time $\phi(\varepsilon)$ across $\Omega_{2}$ cannot be calculated precisely. Nevertheless, for enough small $\varepsilon$, it can be regarded as $g=0$. From (19), it can be obtained that $s=x_{1}$. Integrating both sides of (33) yields

$$
\int_{-\varepsilon^{\eta_{1} /\left(1-\eta_{1}\right)}}^{\varepsilon^{\eta_{1} /\left(1-\eta_{1}\right)}} \mathrm{d} g=\int_{0}^{\phi(\varepsilon)}\left|\frac{\pi \eta_{1}}{2 \beta \varepsilon}\left(\alpha_{2} \operatorname{sig}(s)^{r_{2}}+c_{2} \operatorname{sig}(s)^{\varphi_{2}}\right)\right| \mathrm{d} t .
$$

Solving (34) obtains 


$$
\phi(\varepsilon)=\frac{4 \beta \varepsilon^{1 /\left(1-\eta_{1}\right)}}{\pi \eta_{1}\left(\alpha_{2}\left|x_{1}\right|^{r_{2}}+c_{2}\left|x_{1}\right|^{\varphi_{2}}\right)},
$$

where $1 /\left(1-\eta_{1}\right)>1$. This means that $\phi(\varepsilon)$ can be small enough by selecting sufficiently small $\varepsilon$. Then, the finite time $\phi(\varepsilon)$ can be ignored for smalle because of the estimation conservativeness of $\mathrm{T}_{1}$.

\section{Adaptive Fixed-Time Control of Robot Manipulators}

4.1. Nonsingular Fixed-Time Sliding Mode Surface. Define $e=q-q_{d}$ and $\dot{e}=\dot{q}-\dot{q}_{d}$. The error equation of system (1) can be expressed as follows:

$$
\ddot{e}=f(e, \dot{e})+b(e, \dot{e}) u+\rho,
$$

where

$$
\begin{aligned}
f(e, \dot{e}) & =-M_{0}^{-1}(q) C_{0}(q, \dot{q}) \dot{q}-M_{0}^{-1}(q) G_{0}(q)-\ddot{q}_{d}, \\
b(e, \dot{e}) & =-M_{0}^{-1}(q) .
\end{aligned}
$$

A vector of novel fast nonsingular fixed-time sliding mode surface (NFNFSM) is constructed as

$$
\begin{aligned}
s & =e+\beta \operatorname{sig}\left(\alpha_{1} \operatorname{sig}(e)^{\gamma_{1}}+\dot{e}\right)^{1 / \eta_{1}} \\
& =e+\beta v(e, \dot{e})
\end{aligned}
$$

where $\beta$ is a diagonal matrix:

$$
\begin{aligned}
\beta_{i} & =c_{1}^{-1 / \eta_{1 i}}, \alpha_{1}>0, c_{1}>0, r_{1}>1,0<\varphi_{1}<1, \\
v(e, \dot{e}) & =\left[v\left(e_{1}, \dot{e}_{1}\right), v\left(e_{2}, \dot{e}_{2}\right), \ldots, v\left(e_{n}, \dot{e}_{n}\right)\right]^{T}, \\
v\left(e_{i}, \dot{e}_{i}\right) & =\operatorname{sig}\left(\alpha_{1} \operatorname{sig}\left(e_{i}\right)^{\gamma_{1 i}}+\dot{e}_{i}\right)^{1 / \eta_{1 i}}, \quad i=1,2 \ldots, n, \\
\gamma_{1 i} & =0.5\left(r_{1}+1\right)+0.5\left(r_{1}-1\right) \operatorname{sign}\left(\left|e_{i}\right|-1\right), \\
\eta_{1 i} & =0.5\left(r_{1}+\varphi_{1}\right)+0.5\left(r_{1}-\varphi_{1}\right) \operatorname{sign}\left(\frac{\left|\alpha_{1} \operatorname{sig}\left(e_{i}\right)^{\gamma_{1 i}}+\dot{e}_{i}\right|}{c_{1}}-1\right) .
\end{aligned}
$$

From (38), $\dot{e}_{i}=-\alpha_{1} \operatorname{sig}\left(e_{i}\right)^{\gamma_{1 i}}-c_{1} \operatorname{sig}\left(e_{i}\right)^{\eta_{1 i}}$ when $s_{i}=0$. Consider the Lyapunov function $V_{2}=0.5 e_{i}^{2}$, and its time derivative is $\dot{V}_{2}=e_{i} \dot{e}_{i}=-\alpha_{1}\left|e_{i}\right|^{\gamma_{1 i}+1}-c_{1}\left|e_{i}\right|^{\eta_{1 i}+1}$. When $\left|e_{i}\right| \geq 1$, there is $\dot{V}_{2} \leq-\alpha_{1}\left(2 V_{2}\right)^{\left(r_{1}+1\right) / 2}-c_{1}\left(2 V_{2}\right)^{\left(r_{1}+1\right) / 2}$. When $\left|e_{i}\right|<$ 1 , there is $\dot{V}_{2} \leq-\alpha_{1}\left(2 V_{2}\right)-c_{1}\left(2 V_{2}\right)^{\left(\varphi_{1}+1\right) / 2}$. According to Lemma 3 , the system states can reach the designed sliding surface within a fixed time, given by

$$
T_{s} \leq \frac{2}{\left(2^{\left(r_{1}+1\right) / 2} \alpha_{1}+2^{\left(r_{1}+1\right) / 2} c_{1}\right)\left(r_{1}-1\right)}+\frac{1}{\alpha_{1}\left(1-\varphi_{1}\right)} \ln \left(1+\frac{2 \alpha_{1}}{2^{\left(\varphi_{1}+1\right) / 2} c_{1}}\right) .
$$

4.2. Controller Design. To achieve the fixed-time tracking, an $u=u_{0}+u_{1}+u_{2}$ adaptive NFNFSM controller (ANFNFSMC) is designed as

with

$$
\begin{aligned}
u_{0}= & C_{0}(q, \dot{q}) \dot{q}+G_{0}(q)+M_{0}(q) \ddot{q}_{d}, \\
u_{1}= & -M_{0}(q)\left[\beta^{-1} \eta_{1} \operatorname{sig}\left(\alpha_{1} \operatorname{sig}(e)^{\gamma_{1}}+\dot{e}\right)^{2-\left(1 / \eta_{1}\right)}\right. \\
& +\operatorname{diag}\left(\alpha_{1} \eta_{1 i}\left|e_{i}\right|^{\gamma_{1 i}-1}\right)\left(\alpha_{1} \operatorname{sig}(e)^{\gamma_{1}}+\dot{e}\right) \\
& \left.+\operatorname{diag}\left(\alpha_{1} \gamma_{1 i}\left|e_{i}\right|^{\gamma_{1 i}-1}\right) \dot{e}+\beta^{-1} \operatorname{diag}\left(\eta_{1 i}\left|\alpha_{1} \operatorname{sig}\left(e_{i}\right)^{\gamma_{1 i}}+\dot{e}_{i}\right|^{1-\left(1 / \eta_{1 i}\right.}\right)\right) \\
& \left.\cdot \operatorname{diag}\left(\psi_{\varepsilon}\left(\left|\alpha_{1} \operatorname{sig}\left(e_{i}\right)^{\gamma_{1 i}}+\dot{e}_{i}\right|^{\left(1 / \eta_{1 i}\right)-1}\right)\right) \cdot\left(\alpha_{2} \operatorname{sig}(s)^{r_{2}}+c_{2} \operatorname{sig}(s)^{\varphi_{2}}\right)\right] \\
u_{2}= & -M_{0}(q)\left(\widehat{a}_{0}+\widehat{a}_{1}\|q\|+\widehat{a}_{2}\|\dot{q}\|^{2}\right) \frac{\bar{s}}{\|\bar{s}\|}
\end{aligned}
$$


in which $\alpha_{2}, c_{2}>0, r_{2}>1,0<\varphi_{2}<1, \bar{s}=\left(s^{T} \vartheta\right)^{T}=\vartheta^{T} s$, $\vartheta=\operatorname{diag}\left(\vartheta_{1}, \vartheta_{2}, \ldots, \vartheta_{n}\right), \vartheta_{i}=\left(\beta_{i} / \eta_{1 i}\right)\left|\alpha_{1} \operatorname{sig}\left(e_{i}\right)^{\gamma_{1 i}}+\dot{e}_{i}\right|^{1 / \eta_{1 i}-1}$.

$\hat{a}_{i}$ is the estimation of $a_{i}$, and the adaptive laws are given by

$$
\begin{aligned}
& \dot{\hat{a}}_{0}=\|\bar{s}\|, \\
& \dot{\hat{a}}_{1}=\|\bar{s}\|\|q\|, \\
& \dot{\hat{a}}_{2}=\|\bar{s}\|\|\dot{q}\|^{2} .
\end{aligned}
$$

4.3. Stability Analysis. The fixed-time stability of the error system (36) in both the reaching phase and the sliding phase is stated in Theorem 2 .

Theorem 2. For the error system (36), using the proposed sliding mode surface given by (38) and the novel adaptive nonsingular fixed-time controller defined by (41)-(45), the following performance of the closed-loop system can be achieved:

(1) The variables $s, \widetilde{a}_{0}, \widetilde{a}_{1}$, and $\widetilde{a}_{2}$ are all bounded

(2) The proposed sliding mode manifold scan converge to the neighborhood of $s=0$ within a fixed time

(3) The state variables $e_{i}$ and $\dot{e}_{i}$ can converge into a small region near the origin within a fixed time

Proof. (1) Denote $\widetilde{a}_{i}=a_{i}-\widehat{a}_{i}, i=0,1,2$ and choose a positive Lyapunov function as

$$
V_{3}=0.5\left(s^{T} s+\widetilde{a}_{0}^{2}+\widetilde{a}_{1}^{2}+\widetilde{a}_{2}^{2}\right) .
$$

Differentiating $V_{3}$ with respect to time and taking into account (38), (41)-(45), we obtain

$$
\begin{aligned}
& \dot{V}_{3}=-s^{T} \operatorname{diag}\left(\psi_{\varepsilon}\left(\left|\alpha_{1} \operatorname{sig}\left(e_{i}\right)^{\gamma_{1 i}}+\dot{e}_{i}\right|^{\left(1 / \eta_{1 i}\right)-1}\right)\right)\left(\alpha_{2} \operatorname{sig}(s)^{r_{2}}+c_{2} \operatorname{sig}(s)^{\varphi_{2}}\right) \\
& -s^{T} \operatorname{diag}\left(\alpha_{1}\left|e_{i}\right|^{\gamma_{1 i}-1}\right) s+s^{T}\left(\rho-\left(\widehat{a}_{0}+\widehat{a}_{1}\|q\|+\widehat{a}_{2}\|\dot{q}\|^{2}\right) \frac{\bar{s}}{\|\bar{s}\|}\right) \\
& -\left(a_{0}-\widehat{a}_{0}\right) \dot{\hat{a}}_{0}-\left(a_{1}-\widehat{a}_{1}\right) \dot{\hat{a}}_{1}-\left(a_{2}-\widehat{a}_{2}\right) \dot{\hat{a}}_{2} \\
& \leq-s^{T} \operatorname{diag}\left(\psi_{\varepsilon}\left(\left|\alpha_{1} \operatorname{sig}\left(e_{i}\right)^{\gamma_{1 i}}+\dot{e}_{i}\right|^{\left(1 / \eta_{1 i}\right)-1}\right)\right)\left(\alpha_{2} \operatorname{sig}(s)^{r_{2}}+c_{2} \operatorname{sig}(s)^{\varphi_{2}}\right) \\
& +\|\bar{s}\|\left(\|\rho\|-\left(\widehat{a}_{0}+\widehat{a}_{1}\|q\|+\widehat{a}_{2}\|\dot{q}\|^{2}\right)\right)-\left(a_{0}-\widehat{a}_{0}\right)\|\bar{s}\| \\
& -\left(a_{1}-\widehat{a}_{1}\right)\|\bar{s}\|\|q\|-\left(a_{2}-\widehat{a}_{2}\right)\|\bar{s}\|\|\dot{q}\|^{2} \\
& \leq-s^{T} \operatorname{diag}\left(\psi_{\varepsilon}\left(\left|\alpha_{1} \operatorname{sig}\left(e_{i}\right)^{\gamma_{1 i}}+\dot{e}_{i}\right|^{\left(1 / \eta_{1 i}\right)-1}\right)\right)\left(\alpha_{2} \operatorname{sig}(s)^{r_{2}}+c_{2} \operatorname{sig}(s)^{\varphi_{2}}\right) \\
& +\|\bar{s}\|\left(\|\rho\|-\left(a_{0}+a_{1}\|q\|+a_{2}\|\dot{q}\|^{2}\right)\right) \text {. }
\end{aligned}
$$

According to Assumption 2, there is

$$
\dot{V}_{3} \leq-s^{T} \operatorname{diag}\left(\psi_{\varepsilon}\left(\left|\alpha_{1} \operatorname{sig}\left(e_{i}\right)^{\gamma_{1 i}}+\dot{e}_{i}\right|^{\left(1 / \eta_{1 i}\right)-1}\right)\right) \cdot\left(\alpha_{2} \operatorname{sig}(s)^{r_{2}}+c_{2} \operatorname{sig}(s)^{\varphi_{2}}\right) .
$$

From (48), we know that $\dot{V}_{3} \leq 0$, which implies that $V_{3}$ is bounded. Then, $s, \widetilde{a}_{0}, \widetilde{a}_{1}$, and $\widetilde{a}_{2}$ are all bounded. It can be noted from (38) that the boundedness of $e_{i}, \dot{e}_{i}$ are guaranteed. And according to Assumption 3, it can be known that both $\|q\|$ and $\|\dot{q}\|$ are bounded. Thus, there exists a positive constant $\sigma_{0}$ such that $\|\bar{s}\|\left(\widetilde{a}_{0}+\widetilde{a}_{1}\|q\|+\widetilde{a}_{2}\|\dot{q}\|^{2}\right) \leq \sigma_{0}$.
(2) To examine the fixed-time stability, consider the following positive definite Lyapunov function:

$$
V_{4}=0.5 s^{T} s
$$

Taking the derivative of $V_{4}$ results in 


$$
\begin{aligned}
\dot{V}_{4} \leq & -s^{T} \operatorname{diag}\left(\psi_{\varepsilon}\left(\left|\alpha_{1} \operatorname{sig}\left(e_{i}\right)^{\gamma_{1 i}}+\dot{e}_{i}\right|^{\left(1 / \eta_{1 i}\right)-1}\right)\right)\left(\alpha_{2} \operatorname{sig}(s)^{r_{2}}+c_{2} \operatorname{sig}(s)^{\varphi_{2}}\right) \\
& -s^{T} \operatorname{diag}\left(\alpha_{1}\left|e_{i}\right|^{\gamma_{1 i}-1}\right) s+\|\bar{s}\|\left(\|\rho\|-\left(\widehat{a}_{0}+\widehat{a}_{1}\|q\|+\widehat{a}_{2}\|\dot{q}\|^{2}\right)\right) \\
\leq & -s^{T} \operatorname{diag}\left(\psi_{\varepsilon}\left(\left|\alpha_{1} \operatorname{sig}\left(e_{i}\right)^{\gamma_{1 i}}+\dot{e}_{i}\right|^{\left(1 / \eta_{1 i}\right)-1}\right)\right)\left(\alpha_{2} \operatorname{sig}(s)^{r_{2}}+c_{2} \operatorname{sig}(s)^{\varphi_{2}}\right) \\
& +\|\bar{s}\|\left(a_{0}+a_{1}\|q\|+a_{2}\|\dot{q}\|^{2}\right)-\|\bar{s}\|\left(\widehat{a}_{0}+\widehat{a}_{1}\|q\|+\widehat{a}_{2}\|\dot{q}\|^{2}\right) \\
= & -s^{T} \operatorname{diag}\left(\psi_{\varepsilon}\left(\left|\alpha_{1} \operatorname{sig}\left(e_{i}\right)^{\gamma_{1 i}}+\dot{e}_{i}\right|\left(1 / \eta_{1 i}\right)-1\right)\right)\left(\alpha_{2} \operatorname{sig}(s)^{r_{2}}+c_{2} \operatorname{sig}(s)^{\varphi_{2}}\right) \\
& +\|\bar{s}\|\left(\tilde{a}_{0}+\tilde{a}_{1}\|q\|+\tilde{a}_{2}\|\dot{q}\|^{2}\right) .
\end{aligned}
$$

Based on the above analysis, we know that $s, \widetilde{a}_{0}, \widetilde{a}_{1}$, $\tilde{a}_{2},\|q\|$, and $\|\dot{q}\|$ are all bounded; thus, there exists a positive constant $\sigma$ satisfying that $\|\bar{s}\|\left(\widetilde{a}_{0}+\tilde{a}_{1}\|q\|+\right.$
$\left.\tilde{a}_{2}\|\dot{q}\|^{2}\right)+s^{T}\left[I_{2}-\operatorname{diag}\left(\psi_{\varepsilon}\left(\left|\alpha_{1 i} \operatorname{sig}\left(e_{i}\right)^{\gamma_{1 i}}+\dot{e}_{i}\right|^{1 / \eta_{1 i}-1}\right)\right)\right]$ $\left(\alpha_{2} \operatorname{sig}(s)^{r_{2}}+c_{2} \operatorname{sig}(s)^{\varphi_{2}}\right) \leq \sigma$. Then, according to Lemma 2, (50) is simplified as

$$
\dot{V}_{4} \leq-s^{T}\left(\alpha_{2} \operatorname{sig}(s)^{r_{2}}+c_{2} \operatorname{sig}(s)^{\varphi_{2}}\right)+\sigma \leq-n^{\left(1-r_{2}\right) / 2} \alpha_{2}\left(2 V_{4}\right)^{\left(r_{2}+1\right) / 2}-c_{2}\left(2 V_{4}\right)^{\left(\varphi_{2}+1\right) / 2}+\sigma .
$$

Applying Lemma 1, the system is fixed-time stable with the following convergence region:

$$
\begin{aligned}
\Delta & =\lim _{t \longrightarrow T_{r}} s \mid V_{2} \leq \\
& \min \left\{\left(\frac{\sigma}{2^{\left(r_{2}+1\right) / 2} n^{\left(1-r_{2}\right) / 2} \alpha_{2}(1-\theta)}\right)^{2 /\left(r_{2}+1\right)},\left(\frac{\sigma}{2^{\left(\varphi_{2}+1\right) / 2} c_{2}(1-\theta)}\right)^{2 /\left(\varphi_{2}+1\right)}\right\} .
\end{aligned}
$$

According to Lemma 1, the convergence time of the reaching motion can be estimated as

$$
T_{r} \leq \frac{2}{2^{\left(r_{2}+1\right) / 2} n^{\left(1-r_{2}\right) / 2} \alpha_{2} \theta\left(r_{2}-1\right)}+\frac{2}{2^{\left(\varphi_{2}+1\right) / 2} c_{2} \theta\left(1-\varphi_{2}\right)} .
$$

(3) Once the sliding variable $s_{i}$ converges to the region $\left|s_{i}\right| \leq \Delta, i=1,2, \ldots, n$, there is

$$
s_{i}=e_{i}+\beta_{i} \operatorname{sig}\left(\alpha_{1} \operatorname{sig}\left(e_{i}\right)^{\gamma_{1 i}}+\dot{e}_{i}\right)^{1 / \eta_{1 i}}, \quad\left|s_{i}\right| \leq \Delta .
$$

Equation (54) can be rewritten as

$$
e_{i}+\left(\beta_{i}-\frac{s_{i}}{\operatorname{sig}\left(\alpha_{1} \operatorname{sig}\left(e_{i}\right)^{\gamma_{1 i}}+\dot{e}_{i}\right)^{1 / \eta_{1 i}}}\right) \cdot \operatorname{sig}\left(\alpha_{1} \operatorname{sig}\left(e_{i}\right)^{\gamma_{1 i}}+\dot{e}_{i}\right)^{1 / \eta_{1 i}}=0
$$

When $\quad \beta_{i}-s_{i} / \operatorname{sig}\left(\alpha_{1} \operatorname{sig}\left(e_{i}\right)^{\gamma_{1 i}}+\dot{e}_{i}\right)^{1 / \eta_{1 i}}>0, \quad$ (55) still maintains the form of NFNFSM as (38). Thus, the system trajectory will persistently converge to the NFNFSM (38) until it satisfies the following condition:

$$
\left|\alpha_{1} \operatorname{sig}\left(e_{i}\right)^{\gamma_{1 i}}+\dot{e}_{i}\right|^{1 / \eta_{1 i}} \leq \frac{\Delta}{\beta_{i}}
$$

From (38) and (56), the tracking error will converge to the region

$$
\left|e_{i}\right| \leq \beta_{i}\left|\alpha_{1} \operatorname{sig}\left(e_{i}\right)^{\gamma_{1 i}}+\dot{e}_{i}\right|^{1 / \eta_{1 i}}+\left|s_{i}\right| \leq 2 \Delta
$$

From (56), we have

$$
|| \alpha_{1} \operatorname{sig}\left(e_{i}\right)^{\gamma_{1 i}}|-| \dot{e}_{i}|| \leq\left|\alpha_{1} \operatorname{sig}\left(e_{i}\right)^{\gamma_{1 i}}+\dot{e}_{i}\right| \leq\left(\frac{\Delta}{\beta_{i}}\right)^{\eta_{1 i}} \text {. }
$$

Then,

$$
\left|\alpha_{1} \operatorname{sig}\left(e_{i}\right)^{\gamma_{1 i}}\right|-\left|\dot{e}_{i}\right| \geq-\left(\frac{\Delta}{\beta_{i}}\right)^{\eta_{1 i}}
$$

Thus, the fixed-time convergence region of $e_{i}$ is

$$
\left|\dot{e}_{i}\right| \leq\left|\alpha_{1} \operatorname{sig}\left(e_{i}\right)^{\gamma_{1 i}}\right|+\left(\frac{\Delta}{\beta_{i}}\right)^{\eta_{1 i}} \leq \alpha_{1}(2 \Delta)^{\gamma_{1 i}}+\left(\frac{\Delta}{\beta_{i}}\right)^{\eta_{1 i}} \text {. }
$$

It is concluded that the system states will converge to the set $R=\left\{\left(e_{i}, \dot{e}_{i}\right):\left|e_{i}\right| \leq 2 \Delta,\left|\dot{e}_{i}\right| \leq \alpha_{1}(2 \Delta)^{\gamma_{1 i}}+\left(\Delta / \beta_{i}\right)^{\eta_{1 i}}\right\}$ in fixed time.

Accordingly, the upper bound of the total convergence time can be estimated as 


$$
T=T_{r}+T_{s} \leq \frac{1}{2^{\left(r_{2}-1\right) / 2} n^{\left(1-r_{2}\right) / 2} \alpha_{2} \theta\left(r_{2}-1\right)}+\frac{1}{2^{\left(\varphi_{2}-1\right) / 2} c_{2} \theta\left(1-\varphi_{2}\right)}+\frac{1}{2^{\left(r_{1}-1\right) / 2}\left(\alpha_{1}+c_{1}\right)\left(r_{1}-1\right)}+\frac{1}{\alpha_{1}\left(1-\varphi_{1}\right)} \ln \left(1+\frac{\alpha_{1}}{2^{\left(\varphi_{1}-1\right) / 2} c_{1}}\right) .
$$

The proof of Theorem 2 is completed.

\section{Simulation Study}

In this section, based on MATLAB (R2014a)/Simulink, simulations are carried out to confirm the effectiveness and superiority of the proposed ANFNFSMC method.

The dynamics of a typical two-link robot manipulator can be expressed as [1]

$$
\begin{aligned}
M(q) & =\left[\begin{array}{cc}
N_{1}+2 N_{2} \cos \left(q_{2}\right) & N_{3}+N_{2} \cos \left(q_{2}\right) \\
N_{3}+N_{2} \cos \left(q_{2}\right) & N_{4}
\end{array}\right], \\
C(q, \dot{q}) & =\left[\begin{array}{cc}
-N_{2} \sin \left(q_{2}\right) \dot{q}_{1} & -2 N_{2} \sin \left(q_{2}\right) \dot{q}_{1} \\
0 & N_{2} \sin \left(q_{2}\right) \dot{q}_{2}
\end{array}\right], \\
G(q) & =\left[\begin{array}{c}
N_{5} \cos \left(q_{1}\right)+N_{6} \cos \left(q_{1}+q_{2}\right) \\
N_{6} \cos \left(q_{1}+q_{2}\right)
\end{array}\right],
\end{aligned}
$$

with

$$
\begin{aligned}
& N_{1}=\left(m_{1}+m_{2}\right) l_{1}^{2}+m_{2} l_{2}^{2}+J_{1}, \\
& N_{2}=m_{2} l_{1} l_{2}, \\
& N_{3}=m_{2} l_{2}^{2}, \\
& N_{4}=N_{3}+J_{2}, \\
& N_{5}=\left(m_{1}+m_{2}\right) g l_{1}, \\
& N_{6}=m_{2} g l_{2} .
\end{aligned}
$$

The system parameters in (63) are listed as $m_{1}=0.5 \mathrm{~kg}$, $m_{2}=1.5 \mathrm{~kg}, \quad l_{1}=1 \mathrm{~m}, \quad l_{2}=0.8 \mathrm{~m}, \quad J_{1}=5 \mathrm{~kg} \cdot \mathrm{m}^{2}$, $J_{2}=5 \mathrm{~kg} \cdot \mathrm{m}^{2}, g=9.8 \mathrm{~N} / \mathrm{s}^{2}$. The normal values of $m_{1}$ and $m_{2}$ are set as $m_{10}=0.4 \mathrm{~kg}$ and $m_{20}=1.2 \mathrm{~kg}$. The external disturbances are assumed as $d=[5 \cos (2 t)+2 \sin (2 \pi t), 3$ $\sin (2 t)+2 \sin (\pi t)]^{T}$.

The controller and adaptive law parameters in (43) are set as $\alpha_{1}=c_{1}=1, r_{1}=2, \varphi_{1}=0.6, \alpha_{2}=c_{2}=2, r_{2}=2.5$, $\varphi_{2}=0.6$.

5.1. Tracking Control with Different Initial States. In this case, to confirm the fixed-time tracking performance of the proposed control approach, simulations are performed for trajectory tracking of a robot manipulator with different initial states, as shown in Figures 2-4. The desired signals are $q_{1 d}=0.5 e^{-4 t}-1.2 e^{-t}+1.25$ and $q_{2 d}=e^{-t}-0.5 e^{-4 t}+1.25$. These different initial states are given by

$$
\begin{aligned}
& \text { I1: } q(0)=[1,1]^{T}, \dot{q}(0)=[0,0]^{T}, \\
& \text { I2: } q(0)=[2.5,-0.5]^{T}, \dot{q}(0)=[0,0]^{T}, \\
& \text { I3: } q(0)=[6,5]^{T}, \dot{q}(0)=[0,0]^{T}, \\
& \text { I4: } q(0)=[-2,3]^{T}, \dot{q}(0)=[0,0]^{T} .
\end{aligned}
$$

According to Theorem 2, the system can be stabilized with a unique bounded time for all initial conditions. It is seen from Figure 2 that the total convergence time of the proposed ANFNFSMC with four different initial conditions is bounded by $2 \mathrm{~s}$, which is in line with Theorem 2 . This means that the upper bounded convergence time of the proposed control scheme can be estimated without relying on the initial states and robot model. Tracking error signals are displayed in Figure 3. Figure 4 exhibits the time responses of the proposed sliding surface. It is observed from Figure 4 that the reaching time under the proposed ANFNFSMC for all different initial conditions are upper bounded by $1 \mathrm{~s}$, which do not exceed the theoretical maximum in Theorem 2. Simulation results show that the tracking errors and the sliding variables can converge to the equilibrium within a fixed time, which implies that the upper bound of convergence time is only related to the design parameters and can be acquired in advance.

5.2. Tracking Control with Different Desired Signals. In this case, the proposed ANFNFSMC method is employed in the trajectory tracking of a robot manipulator with different desired reference signals. The initial conditions are set as $q(0)=[1.5,1]^{T}, \dot{q}(0)=[0,0]^{T}$. These different desired signals $q_{d}=\left[q_{1 d}, q_{2 d}\right]^{T}$ are given by

$$
\begin{aligned}
& \text { D1: } q_{1 d}=0.5 e^{-4 t}-1.2 e^{-t}+1.25, q_{2 d}=e^{-t}-0.5 e^{-4 t}+1.25, \\
& \text { D2: } q_{1 d}=0.5 e^{-4 t}-1.2 e^{-t}+2, q_{2 d}=e^{-t}-0.5 e^{-4 t}+2, \\
& \text { D3: } q_{1 d}=0.5 e^{-4 t}-1.2 e^{-t}+3, q_{2 d}=e^{-t}-0.5 e^{-4 t}+3, \\
& \text { D4: } q_{1 d}=0.5 e^{-4 t}-1.2 e^{-t}+5, q_{2 d}=e^{-t}-0.5 e^{-4 t}+5 .
\end{aligned}
$$

The simulation results with different desired reference signals under the proposed control scheme are displayed in Figures 5-7. The positions of joints 1 and 2 are shown in Figure 5. Figure 6 illustrates the tracking error signals. The time responses of the proposed nonsingular fixed-time sliding surfaces are depicted in Figure 7. Observed by Figures 5-7, the proposed ANFNFSMC scheme can guarantee the robot manipulator's track of different desired reference trajectories with a bounded time. Since the uncertain lumped disturbances are compensated by using the adaptive technique, the sliding surfaces and the position tracking errors can be stabilized to the equilibrium within a fixed time. The upper bound of the convergence time is independent of desired reference signals and can be known priorly.

5.3. Various Control Parameters. To illustrate the influence of control parameters on the tracking performance of the proposed control method, the simulations with four sets of 


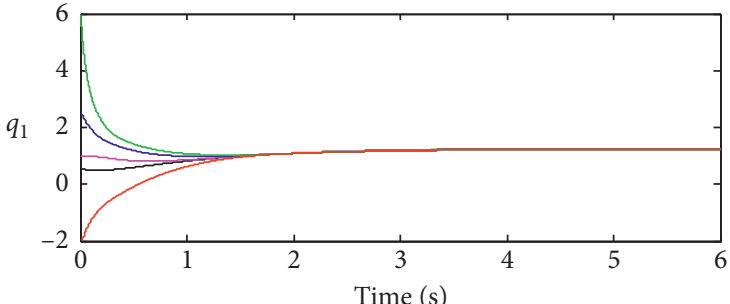

$\begin{array}{ll}\text { - Desired signal } & \text { I3 } \\ \text { I1 } & \text { I4 } \\ \text { I2 } & \end{array}$

(a)

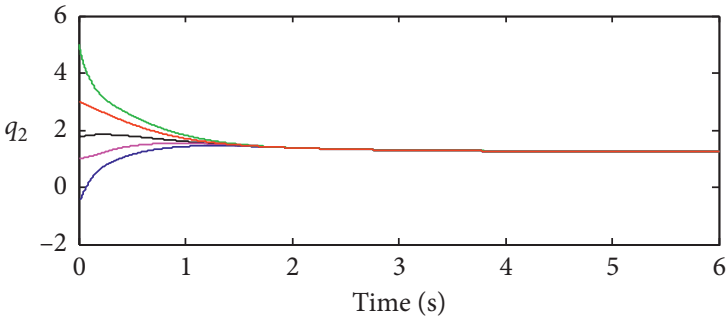

$\begin{array}{ll}\text { Desired signal } & \text { I3 } \\ \text { I1 } & - \text { I4 }\end{array}$

(b)

FIgURE 2: Position tracking performance with different initial states.

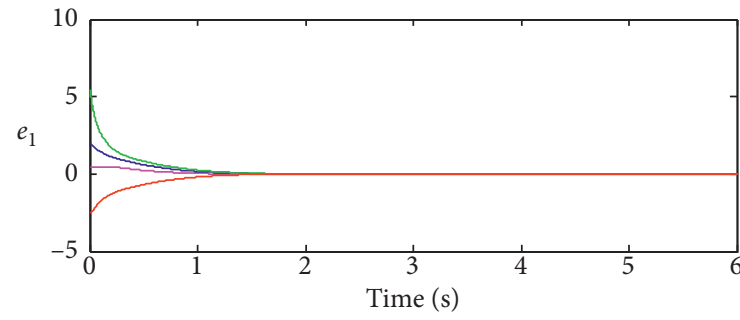

$\begin{array}{ll}\mathrm{I} 1 & -\mathrm{I} 3 \\ \mathrm{I} 2 & -\mathrm{I} 4\end{array}$

(a)

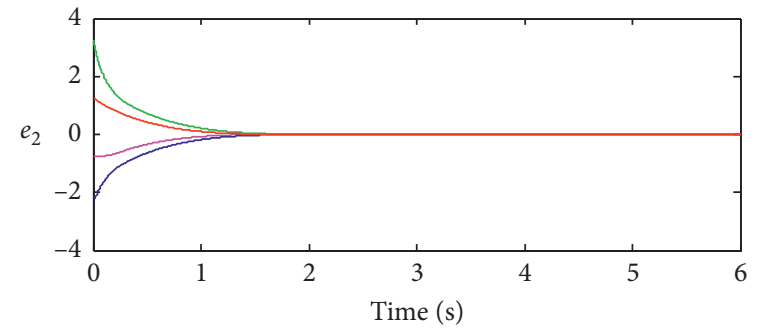

- I1

$-\mathrm{I} 3$

(b)

FIgURE 3: Position tracking errors with different initial states.

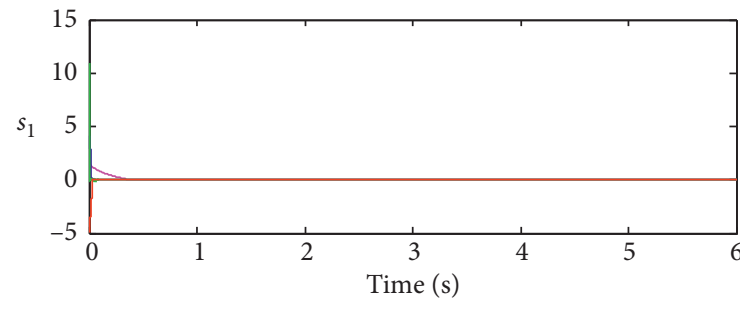

I1
$-\mathrm{I} 2$

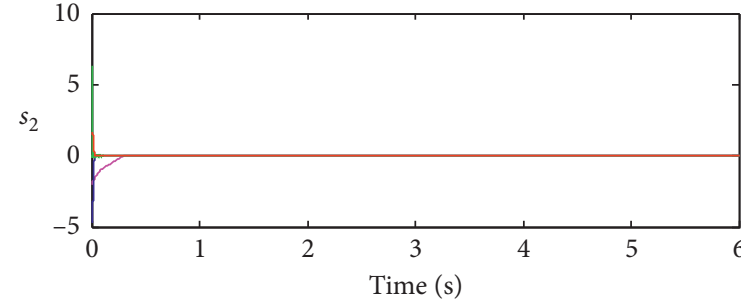

- I1

I2

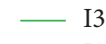

$-\mathrm{I} 4$

(a)

(b)

FIgURE 4: Sliding surfaces versus time with different initial states.

parameters are accomplished in Figures $8-10$. The initial states are set as $q(0)=[1.5,1.5]^{T}, \dot{q}(0)=[0,0]^{T}$. These sets of parameters are listed as follows:

$P 1: \alpha_{1}=c_{1}=1, r_{1}=2, \varphi_{1}=0.6, \alpha_{2}=c_{2}=2, r_{2}=2.5, \varphi_{2}=0.6$,

$P 2: \alpha_{1}=c_{1}=1, r_{1}=2.5, \varphi_{1}=0.55, \alpha_{2}=c_{2}=2, r_{2}=3, \varphi_{2}=0.55$,

P3: $\alpha_{1}=c_{1}=2, r_{1}=2, \varphi_{1}=0.6, \alpha_{2}=c_{2}=2, r_{2}=2.5, \varphi_{2}=0.6$,

P4: $\alpha_{1}=c_{1}=1, r_{1}=2, \varphi_{1}=0.6, \alpha_{2}=c_{2}=8, r_{2}=2.5, \varphi_{2}=0.6$.
As shown in Figures 8-10, the proposed ANFNFSMC with four sets of control parameters have different convergence times that are bounded by their upper bound of the convergence time. The simulation results show that the convergence time is only related to the control parameters, which further confirms the theoretical analysis in Theorem 2. It is noted from Figure 9 that since $\alpha_{1}$ and $c_{1}$ increase, the total convergence time of $\mathrm{P} 3$ is less than those of the other three cases. Given in Figure 10, the reaching time of P4 is shorter than those of the other three cases because $\alpha_{2}$ and 


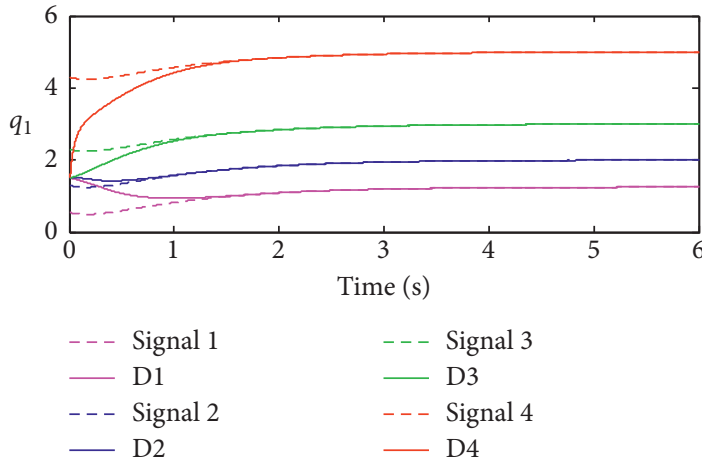

(a)
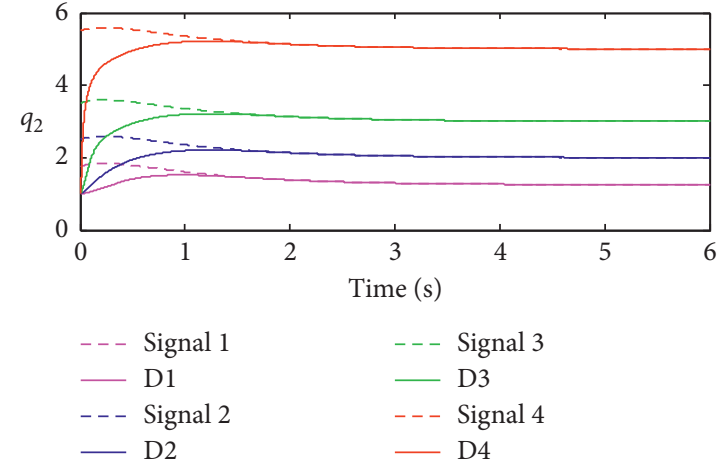

(b)

FIGURE 5: Position tracking performance with different desired signals.

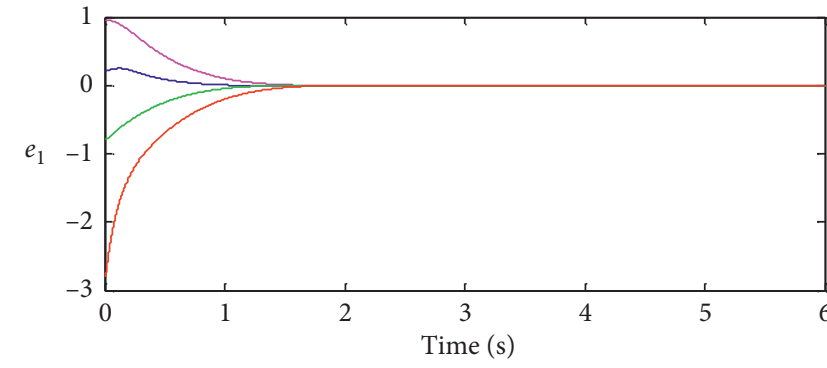

$-\mathrm{D} 1$
$-\mathrm{D} 2$
- D3

- D4

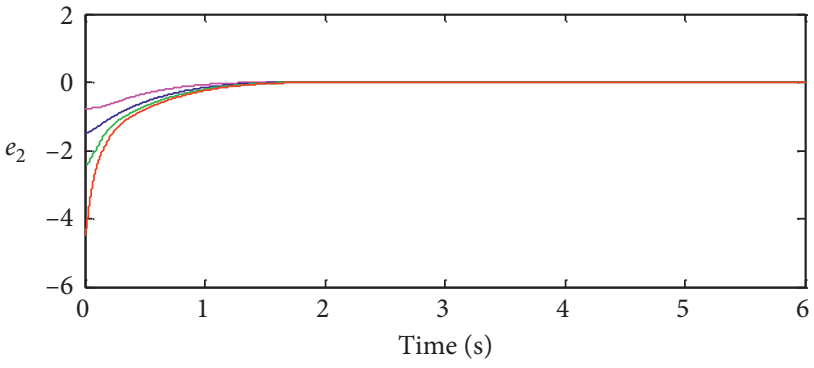

$-\mathrm{D} 1$
- D3

(b)

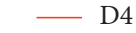

(a)

Figure 6: Position tracking errors with different desired signals.

$c_{2}$ increase. Additionally, it is obvious that the parameters $\alpha_{1}$, $c_{1}, \alpha_{2}$, and $c_{2}$ have more effect on the tracking performance than the parameters $r_{1}, \varphi_{1}, r_{2}$, and $\varphi_{2}$. Although, the parameters $\alpha_{1}, c_{1}, \alpha_{2}$, and $c_{2}$ can be set sufficiently large so that both the convergence regions and convergence time can be as small as desired. However, the design parameters cannot be large enough because of the control saturation constraint. Accordingly, the parameters selection should be considered with a trade-off between the control input and the tracking performance.
5.4. Comparison with Other Control Methods. To show the superiority of the proposed control method, other control methods are also utilized in comparative simulations. The initial conditions are set as $q(0)=[2,0.5]^{T}, \dot{q}(0)=[0,0]^{T}$. The desired reference signals are $q_{1 d}=0.5 e^{-4 t}-1.2 e^{-t}+$ 1.25 and $q_{2 d}=e^{-t}-0.5 e^{-4 t}+1.25$.

Based on the above simulation conditions, comparative simulations are performed with the other three sliding mode control methods.

According to [41], controller 1 is designed as

$$
\begin{aligned}
s & =e+\operatorname{sig}(e)^{\kappa_{1}}+\operatorname{sig}(\dot{e})^{\kappa_{2}}, \\
u & =u_{0}+u_{1}, \\
u_{1} & =-M_{0}(q)\left[\kappa_{2}^{-1}\left(I_{2}+\kappa_{1} \operatorname{diag}\left(|e|^{\kappa_{1}-I_{2}}\right)\right) \cdot \operatorname{sig}(\dot{e})^{2 I_{2}-\kappa_{2}}+\ell_{2} s+\left(\ell_{1}+u_{\text {aux }}\right) \frac{s}{\|s\|}\right], \\
u_{\text {aux }} & =-\left\|M_{0}^{-1}(q)\right\|\left(a_{0}+a_{1}\|q\|+a_{2}\|\dot{q}\|^{2}\right),
\end{aligned}
$$




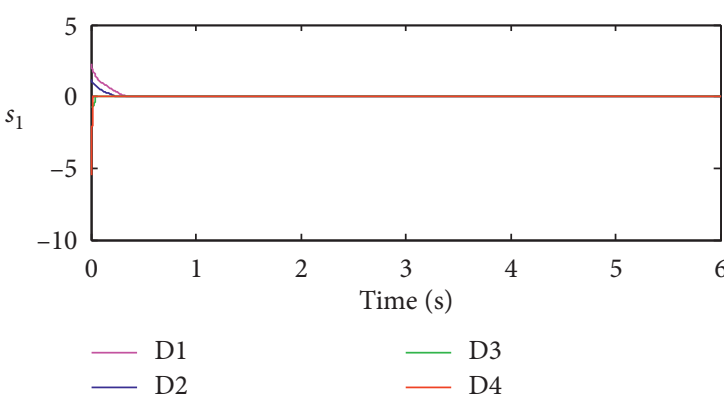

(a)
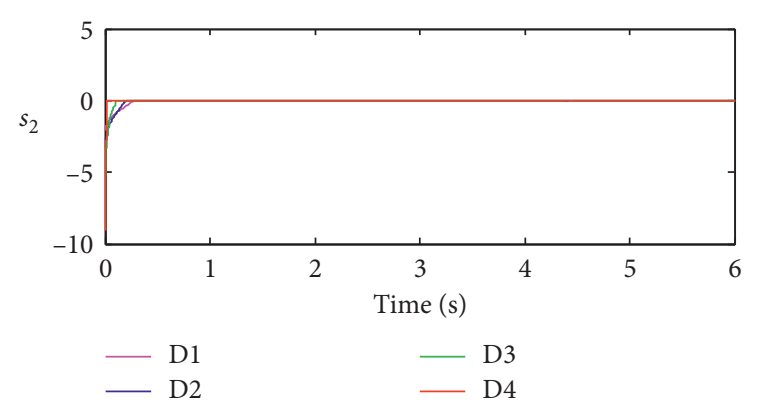

(b)

Figure 7: Sliding surfaces versus time with different desired signals.

where $u_{0}$ is defined as (42). The parameters are chosen as $\kappa_{1}=2, \kappa_{2}=5 / 3, \ell_{2}=2, \ell_{1}=1, a_{0}=12, a_{1}=2.2, a_{2}=2.8$.

According to [9], controller 2 is designed as

$$
\begin{aligned}
\Omega & =\dot{e}+l e, \\
s & =\Gamma\left(\Omega-\Omega(0) e^{-v t}\right), \\
u & =u_{0}+u_{1}+u_{2}, \\
u_{1} & =-M_{0}(q)\left(l \dot{e}+\nu \Omega(0) e^{-v t}\right), \\
u_{2} & =-M_{0}(q)\left(k s \Gamma^{T}+\omega_{0} \operatorname{sig}\left(s \Gamma^{T}\right)^{0.5}+\omega_{1}\right), \\
\dot{\omega}_{1} & =\widehat{\omega} \operatorname{sign}\left(s \Gamma^{T}\right), \\
\dot{\omega} & =\omega_{2}\left\|s \Gamma^{T}\right\|,
\end{aligned}
$$

where $u_{0}$ is defined as (42). The parameters are chosen as $\Gamma=1, l=2, v=4, k=2, \omega_{0}=20, \omega_{2}=1$.

Using the control method presented in [26], controller 3 is designed as

$$
\begin{aligned}
& s=e+\iota_{1} \operatorname{sig}(e)^{\phi_{1}}+\iota_{2} \operatorname{sig}(\dot{e})^{\phi_{2}}, \\
& u=u_{0}+u_{1}+u_{2}, \\
& u_{1}=-\frac{M_{0}(q)}{l_{2} \phi_{2}}|\dot{e}|^{2-\phi_{2}}\left(1+\phi_{1} \iota_{1}|e|^{\phi_{1}-1}\right) \operatorname{sign}(\dot{e}), \\
& u_{2}=-M_{0}(q)\left(\kappa s+\left(\hat{a}_{0}+\widehat{a}_{1}\|q\|+\widehat{a}_{2}\|\dot{q}\|^{2}+\xi\right) \operatorname{sign}(s)\right), \\
& \dot{\vec{a}}_{0}=v_{0}\|s\|\|\dot{e}\|^{\phi_{2}-1}, \\
& \dot{\vec{a}}_{1}=v_{1}\|s\|\|q\|\|\dot{e}\|^{\phi_{2}-1}, \\
& \dot{\vec{a}}_{2}=v_{2}\|s\|\|\dot{q}\|^{2}\|\dot{e}\|^{\phi_{2}-1},
\end{aligned}
$$

where $u_{0}$ is defined as (42). The parameters are chosen as

$$
\begin{aligned}
\iota_{1} & =\iota_{2} \\
\phi_{1} & =2, \\
\phi_{2} & =\frac{5}{3}, \\
\kappa & =2, \\
\xi & =1, \\
v_{0} & =v_{1}=v_{2}=1 .
\end{aligned}
$$

Four controllers are applied for tracking control of the robot manipulator. The position tracking performance and position tracking errors under these four controllers are shown in Figures 11 and 12, respectively. Figures 11 and 12 illustrate the faster convergence of the control system by the proposed controller compared to the other three controllers. The zoomed position tracking errors are plotted in Figure 13. It is seen from Figure 13 that the proposed controller achieves higher steady-state tracking accuracy and smaller fluctuation in comparison with the other three controllers. The comparative simulations make clear that the tracking performance of the proposed fixed-time control method is better than the other three control methods.

For quantitative analysis, two comparison criteria are given as follows: the integral of the absolute value of the error (IAE) defined as IAE $=\int|e| \mathrm{d} t$ and the integral of the time multiplied by the absolute value of the error (ITAE) defined as ITAE $=\int t|e| \mathrm{d} t$. Under these four controllers, the values of two comparison criteria in $t=6 \mathrm{~s}$ are shown in Table 1. It is found from Table 1 that the values of IAE and ITAE for the proposed controller are smaller than the other three controllers, which indicates that the proposed controller has better control performance.

On the basis of all the above simulations and analysis, it can be concluded that the proposed ANFNFSMC method provides excellent tracking performance with regard to fast fixed-time convergence and high steady-state tracking precision. 


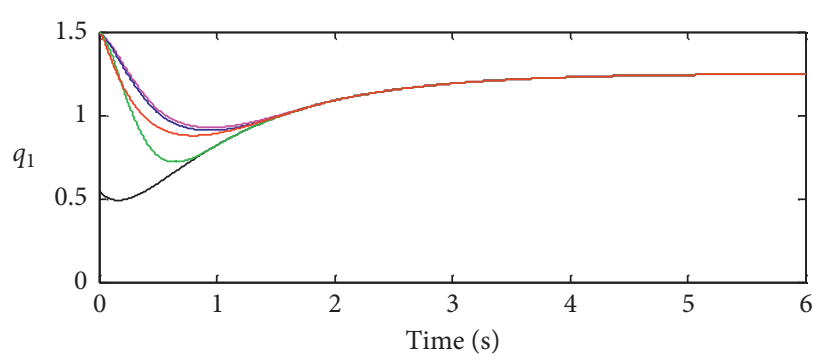

P1
P2
P3

- P4

— Desired signal

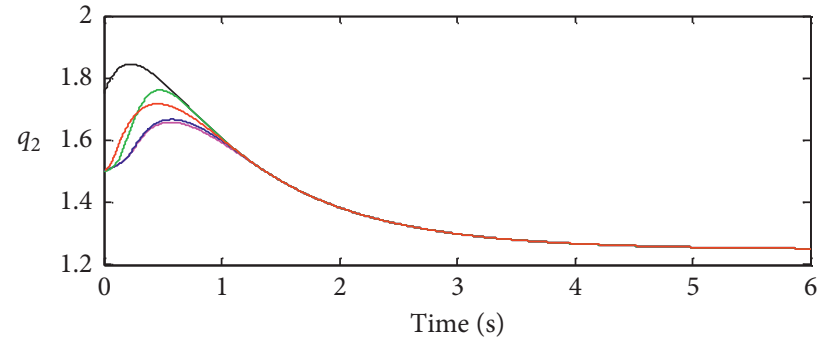

$\mathrm{P} 1$
$-\mathrm{P} 2$

- P3
- P4

- Desired signal

(a)

(b)

FIGURE 8: Position tracking performance with different parameters.

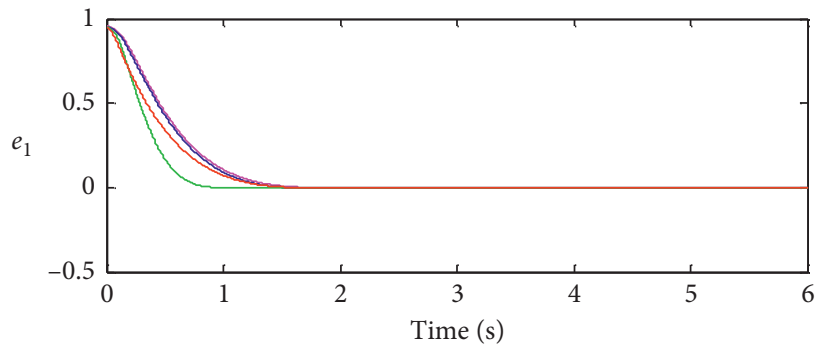

— P1

- P2

- P3

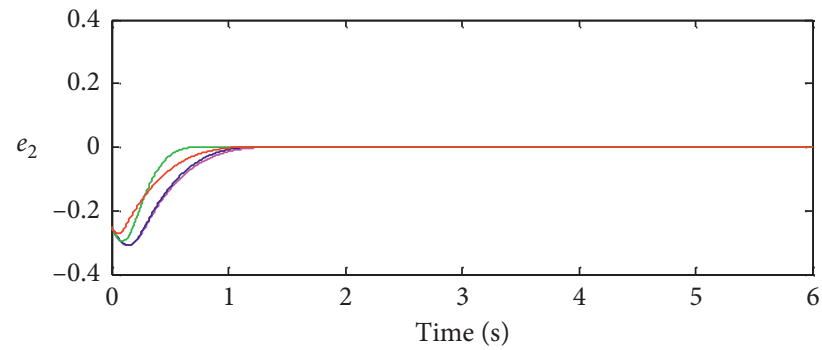

)

P1
$-\mathrm{P} 2$

- P3

(b)

FIgURE 9: Position tracking errors with different parameters.

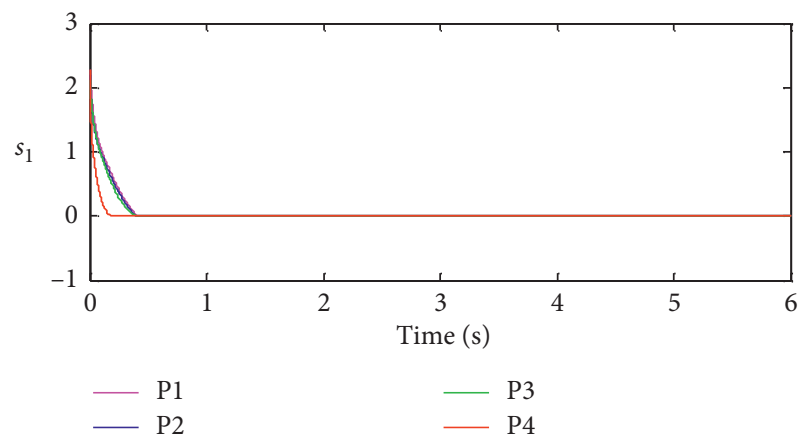

(a)

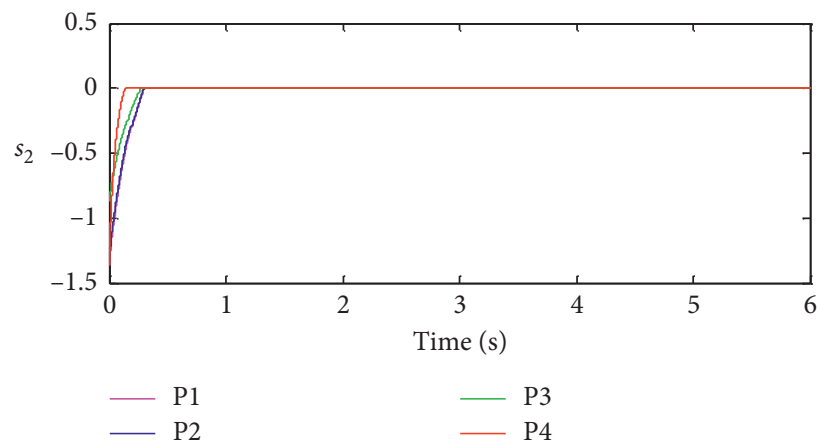

(b)

FIGURE 10: Sliding surfaces versus time with different parameters. 


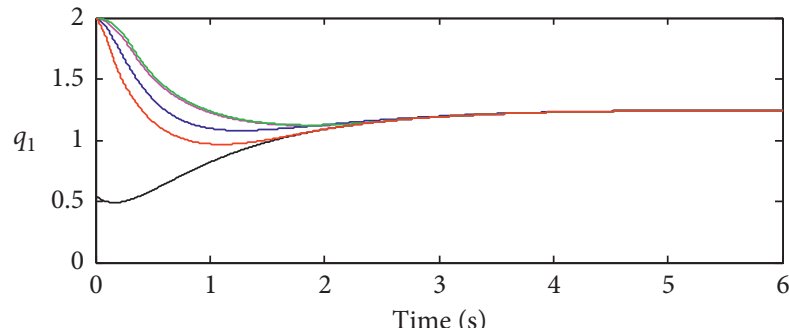

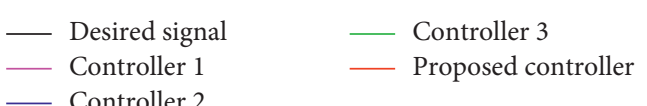

(a)

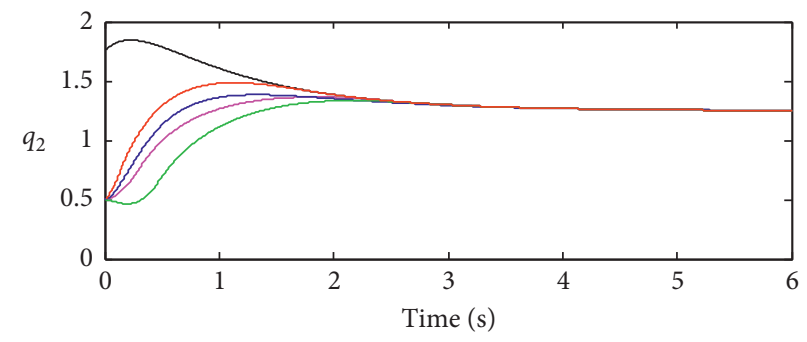

$\begin{array}{ll}\text { — Desired signal } & \text { Controller } 3 \\ \text { Controller } 1 & \text { Proposed controller }\end{array}$

(b)

FIgURe 11: Position tracking performance under four controllers.

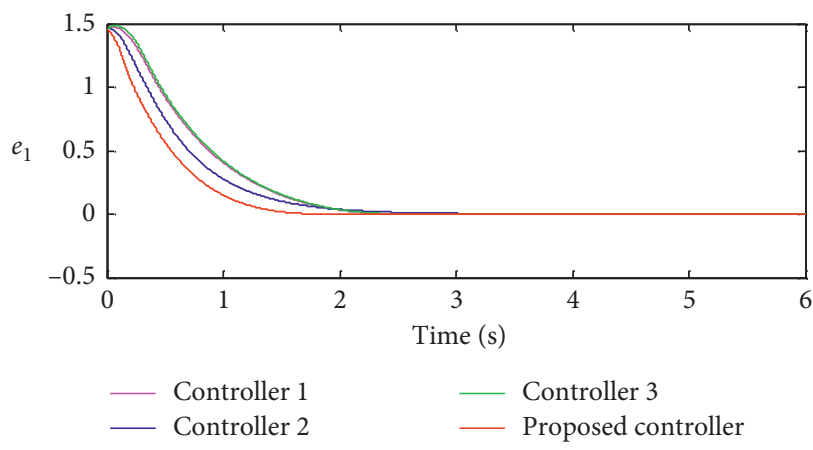

(a)

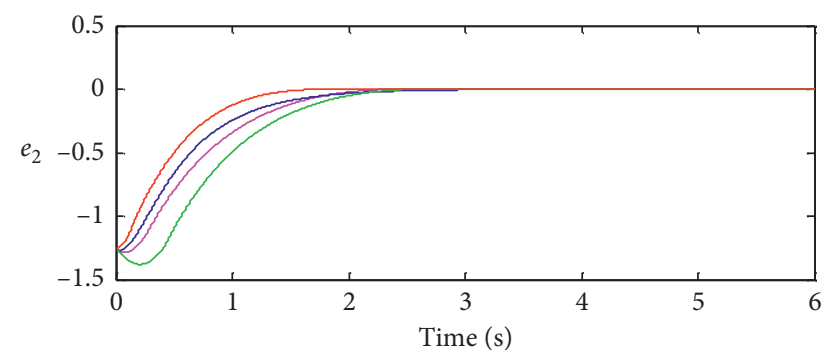

$\begin{array}{ll}\text { Controller } 1 & \text { Controller } 3 \\ \text { Controller } 2 & \text { Proposed controller }\end{array}$

(b)

Figure 12: Position tracking errors under four controllers.

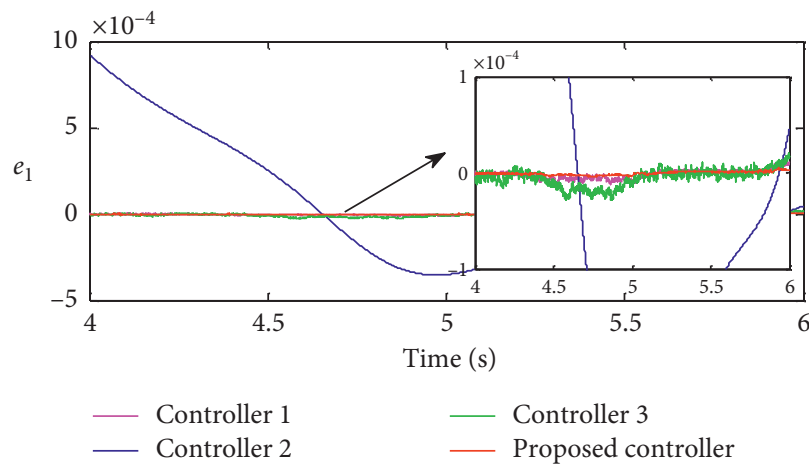

(a)

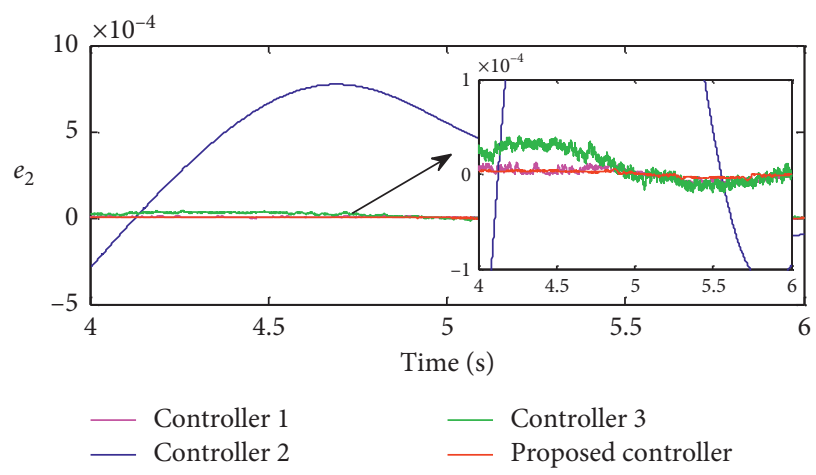

(b)

Figure 13: Zoomed position tracking errors under four controllers.

TABLE 1: Comparison of control performance.

\begin{tabular}{llcr}
\hline Controllers & Joints & IAE & ITAE \\
\hline \multirow{2}{*}{ Controller 1 } & Joint 1 & 1.1377 & 0.6199 \\
& Joint 2 & 0.9725 & 0.5155 \\
\hline \multirow{2}{*}{ Controller 2 } & Joint 1 & 0.9569 & 0.5091 \\
\multirow{2}{*}{ Controller 3 } & Joint 2 & 0.8429 & 0.4496 \\
\hline \multirow{2}{*}{ Proposed controller } & Joint 1 & 1.1660 & 0.6402 \\
& Joint 2 & 1.2657 & 0.7525 \\
\hline
\end{tabular}




\section{Conclusion}

In this paper, the fixed-time tracking control problem of uncertain robot manipulators is researched. The NFNFSM is constructed to circumvent the singularity and achieve fast convergence. Based on the proposed NFNFSM and the adaptive technique, a novel adaptive nonsingular fixed-time sliding mode control method is presented to guarantee the properties of disturbance suppression and fixed-time convergence. The convergence time of the system can be estimated regardless of the initial states, and the system states can converge into a small vicinity of the equilibrium point within a fixed time. Compared with the existing control methods $[9,26,41]$, the proposed control scheme can provide a faster convergence rate and higher control accuracy. Moreover, the control method can be also applicable to some other complicated second-order nonlinear systems. It is noted that the proposed controller design requires the velocity states of robot manipulator systems. However, the velocity states are often not easily obtained or measured. Thus, a velocity-free fixed-time controller design for uncertain robot manipulators will be included in our future investigation.

\section{Data Availability}

The data used to support the findings of this study are available from the corresponding author upon request.

\section{Conflicts of Interest}

The authors declare that they have no conflicts of interest.

\section{Acknowledgments}

This work was supported by the National Natural Science Foundation of China under Grant no. 61703202, the Key Research Development Project of Jiangsu Province under Grant BE2017164, and the Natural Science Foundation of Universities of Anhui Province under Grant no. KJ2020A0692.

\section{References}

[1] Y. Feng, X. Yu, and Z. Man, "Non-singular terminal sliding mode control of rigid manipulators," Automatica, vol. 38, no. 12, pp. 2159-2167, 2002.

[2] L. Wang, T. Chai, and L. Zhai, "Neural-network-based terminal sliding-mode control of robotic manipulators including actuator dynamics," IEEE Transactions on Industrial Electronics, vol. 56, no. 9, pp. 3296-3304, 2009.

[3] W. Zhang, X. Ye, L. Jiang, Y. Zhu, X. Ji, and X. Hu, "Output feedback control for free-floating space robotic manipulators base on adaptive fuzzy neural network," Aerospace Science and Technology, vol. 29, no. 1, pp. 135-143, 2013.

[4] L. Zhang, L. Liu, Z. Wang, and Y. Xia, "Continuous finite-time control for uncertain robot manipulators with integral sliding mode," IET Control Theory \& Applications, vol. 12, no. 11, pp. 1621-1627, 2018.

[5] B. Xiao, L. Cao, S. Xu, and L. Liu, "Robust tracking control of robot manipulators with actuator faults and joint velocity measurement uncertainty," IEEE/ASME Transactions on Mechatronics, vol. 25, no. 3, pp. 1354-1365, 2020.

[6] B. Zhou, L. Yang, C. Wang, Y. Chen, and K. Chen, "Inverse jacobian adaptive tracking control of robot manipulators with kinematic, dynamic, and actuator uncertainties," Complexity, vol. 2020, Article ID 5070354, 12 pages, 2020.

[7] J. Shi, H. Liu, and N. Bajcinca, "Robust control of robotic manipulators based on integral sliding mode," International Journal of Control, vol. 81, no. 10, pp. 1537-1548, 2008.

[8] S. Mondal and C. Mahanta, "Adaptive second order terminal sliding mode controller for robotic manipulators," Journal of the Franklin Institute, vol. 351, no. 4, pp. 2356-2377, 2014.

[9] S. Mobayen, F. Tchier, and L. Ragoub, "Design of an adaptive tracker forn-link rigid robotic manipulators based on supertwisting global nonlinear sliding mode control," International Journal of Systems Science, vol. 48, no. 9, pp. 1990-2002, 2017.

[10] J. Baek, M. Jin, and S. Han, "A new adaptive sliding-mode control scheme for application to robot manipulators," IEEE Transactions on Industrial Electronics, vol. 63, no. 6, pp. 3628-3637, 2016.

[11] C.-S. Jeong, J.-S. Kim, and S.-I. Han, "Tracking error constrained super-twisting sliding mode control for robotic systems," International Journal of Control, Automation and Systems, vol. 16, no. 2, pp. 804-814, 2018.

[12] W. R. Abdul-Adheem, I. K. Ibraheem, J. A. Humaidi, and A. T. Azar, "Model-free active input-output feedback linearization of a single-link flexible joint manipulator: an improved active disturbance rejection control approach," Measurement and Control, vol. 53, pp. 1-6, 2020.

[13] M. Van, M. Mavrovouniotis, and S. Ge, "An adaptive backstepping nonsingular fast terminal sliding mode control for robust fault tolerant control of robot manipulators," IEEE Transactions on Systems, Man, and Cybernetics: Systems, vol. 49, no. 7, pp. 1448-1458, 2019.

[14] W. He and Y. Dong, "Adaptive fuzzy neural network control for a constrained robot using impedance learning," IEEE Transactions on Neural Networks and Learning Systems, vol. 29, no. 4, pp. 1174-1186, 2018.

[15] M. Esfandiari, S. Chan, G. Sutherland, and D. Westwick, "Nonlinear model predictive control of robot manipulators using quasi-LPV representation," in Proceedings of the 7th International Conference on Control, Mechatronics and Automation (ICCMA), Delft, The Netherlands, November 2019.

[16] Y. Wang, Y. Xia, H. Li, and P. Zhou, "A new integral sliding mode design method for nonlinear stochastic systems," Automatica, vol. 90, pp. 304-309, 2018.

[17] H. Pan, G. Zhang, H. Ouyang, and L. Mei, "A novel global fast terminal sliding mode control scheme for second-order systems,” IEEE Access, vol. 8, pp. 22758-22769, 2020.

[18] Y. Wang, H. Pu, P. Shi, C. K. Ahn, and J. Luo, "Sliding mode control for singularly perturbed Markov jump descriptor systems with nonlinear perturbation," Automatica, vol. 127, Article ID 109515, 2021.

[19] S. T. Venkataraman and S. Gulati, "Control of nonlinear systems using terminal sliding modes," Journal of Dynamic Systems, Measurement, and Control, vol. 115, no. 3, pp. 554-560, 1993.

[20] Z. Man, A. P. Paplinski, and H. R. Wu, "A robust MIMO terminal sliding mode control scheme for rigid robotic manipulators," IEEE Transactions on Automatic Control, vol. 39, no. 12, pp. 2464-2469, 1994.

[21] K. B. Park and J. J. Lee, "Comments on 'A robust MIMO terminal sliding mode control scheme for rigid robotic 
manipulators'," IEEE Transactions on Automatic Control, vol. 41, no. 5, pp. 761-762, 1996.

[22] S. Yu, X. Yu, B. Shirinzadeh, and Z. Man, "Continuous finitetime control for robotic manipulators with terminal sliding mode," Automatica, vol. 41, no. 11, pp. 1957-1964, 2005.

[23] A. Al-Ghanimi, J. Zheng, and Z. Man, "Robust and fast nonsingular terminal sliding mode control for piezoelectric actuators," IET Control Theory and Applications, vol. 9, no. 18, pp. 2678-2687, 2015.

[24] Y. Wang, K. Zhu, F. Yan, and B. Chen, "Adaptive supertwisting nonsingular fast terminal sliding mode control for cable-driven manipulators using time-delay estimation," Advances in Engineering Software, vol. 128, pp. 113-124, 2019.

[25] K. Lu and Y. Xia, "Adaptive attitude tracking control for rigid spacecraft with finite-time convergence," Automatica, vol. 49, no. 12, pp. 3591-3599, 2013.

[26] M. Boukattaya, N. Mezghani, and T. Damak, "Adaptive nonsingular fast terminal sliding-mode control for the tracking problem of uncertain dynamical systems," ISA Transactions, vol. 77, pp. 1-19, 2018.

[27] L. Zhang, Y. Su, and Z. Wang, "A simple non-singular terminal sliding mode control for uncertain robot manipulators," in Proceedings of the Institution of Mechanical Engineers, Part I: Journal of Systems and Control Engineering, vol. 233, no. 6, pp. 666-676, 2019.

[28] S. Yi and J. Zhai, "Adaptive second-order fast nonsingular terminal sliding mode control for robotic manipulators," ISA Transactions, vol. 90, pp. 41-51, 2019.

[29] H. Wang, B. Su, Y. Wang, and J. Gao, "Adaptive sliding mode fixed-time tracking control based on fixed-time sliding mode disturbance observer with dead-zone input," Complexity, vol. 2019, Article ID 8951382, 14 pages, 2019.

[30] Z. Cheng, H. Wu, B. Wang, L. Liu, and Y. Wang, "Fixed-time convergent guidance law with impact angle control," Complexity, vol. 2020, Article ID 5019689, 9 pages, 2020.

[31] H. Pan, G. Zhang, H. Ouyang, and L. Mei, "Novel fixed-time nonsingular fast terminal sliding mode control for secondorder uncertain systems based on adaptive disturbance observer," IEEE Access, vol. 8, pp. 126615-126627, 2020.

[32] B. Tian, Z. Zuo, X. Yan, and H. Wang, "A fixed-time output feedback control scheme for double integrator systems," Automatica, vol. 80, pp. 17-24, 2017.

[33] Q. Chen, S. Xie, M. Sun, and X. He, "Adaptive nonsingular fixed-time attitude stabilization of uncertain spacecraft," IEEE Transactions on Aerospace and Electronic Systems, vol. 54, no. 6, pp. 2937-2950, 2018.

[34] Y. Zhang, S. Tang, and J. Guo, “Adaptive terminal angle constraint interception against maneuvering targets with fast fixed-time convergence," International Journal of Robust and Nonlinear Control, vol. 28, no. 1, pp. 2996-3012, 2018.

[35] Y. Tian, Y. Cai, and Y. Deng, "A fast nonsingular terminal sliding mode control method for nonlinear systems with fixed-time stability guarantees," IEEE Access, vol. 8, pp. 60444-60454, 2020.

[36] S. P. Bhat and D. S. Bernstein, "Finite-time stability of continuous autonomous systems," SIAM Journal on Control and Optimization, vol. 38, no. 3, pp. 751-766, 2000.

[37] B. Jiang, Q. Hu, and M. I. Friswell, "Fixed-time attitude control for rigid spacecraft with actuator saturation and faults," IEEE Transactions on Control Systems Technology, vol. 24, no. 5, pp. 1892-1898, 2016.

[38] Y. Huang and Y. Jia, "Adaptive fixed-time six-DOF tracking control for noncooperative spacecraft fly-around mission,"
IEEE Transactions on Control System Technology, vol. 99, pp. 1-9, 2018.

[39] Z. Zuo, "Nonsingular fixed-time consensus tracking for second-order multi-agent networks," Automatica, vol. 54, pp. 305-309, 2015.

[40] H. Li and Y. Cai, "On SFTSM control with fixed-time convergence," IET Control Theory \& Applications, vol. 11, no. 6, pp. 766-773, 2017.

[41] L. Yang and J. Yang, "Nonsingular fast terminal sliding-mode control for nonlinear dynamical systems," International Journal of Robust and Nonlinear Control, vol. 21, no. 16, pp. 1865-1879, 2011. 